\title{
Fluid flow and solute dilution in laser linear butt joining of $304 \mathrm{SS}$ and $\mathrm{Ni}$
}

\author{
Zhiyong Li ${ }^{\mathrm{a}, \mathrm{b}}$, Gang $\mathrm{Yu}^{\mathrm{a}, \mathrm{b}, \mathrm{c}, *}$, Xiuli He $\mathrm{He}^{\mathrm{a}, \mathrm{b}, *}$, Shaoxia $\mathrm{Li}^{\mathrm{a}, \mathrm{b}}$, Zixun $\mathrm{Li}^{\mathrm{a}} \mathrm{b}$ \\ a Key Laboratory of Mechanics in Advanced Manufacturing, Institute of Mechanics, Chinese Academy of Sciences, Beijing 100190, China \\ ${ }^{\mathrm{b}}$ School of Engineering Science, University of Chinese Academy of Sciences, Beijing 100049, China \\ ${ }^{c}$ Center of Materials Science and Optoelectronics Engineering, University of Chinese Academy of Sciences, Beijing 100049, China
}

\section{A R T I C L E I N F O}

\section{Article history:}

Received 24 December 2019

Revised 11 July 2020

Accepted 20 July 2020

Available online 10 August 2020

\section{Keywords:}

Thermal behavior

Fluid flow

Solidification characteristics

Mass transfer

Dissimilar welding

Additive manufacturing

\begin{abstract}
A B S T R A C T
Laser linear welding combined with advanced auto-control technology is a promising joint method of dissimilar metals. In this study, an improved three-dimensional model was developed to investigate the thermal behavior, fluid flow and concentration dilution during laser linear dissimilar welding of 304 stainless steel and nickel. The fluid flow and solidification behavior were presented in a 3D view to deeply study the thermal behavior and convection characteristics inside the melt pool. The relationship between dynamic characteristics and concentration dilution was studied numerically and experimentally, and the effects of welding speed, temperature coefficient of surface tension (TCST) and dynamic viscosity on mass transfer were also investigated. Larger cooling rate (GR) and smaller morphology parameter (G/R) were found at top area of the weld pool (WP). Elements from SS ( $\mathrm{Cr}$ and Fe) were mainly mixed at front side, but Ni was mainly diluted at middle part. Resulting from the Marangoni convection, all the elements were mainly mixed at top area and then transported to the bottom. The redistribution of the species was also observed at each side, resulting in a relatively uniform distribution at each side. Nevertheless, the effects of welding speed, TCST and dynamic viscosity on solute dilution were simulated and proved non-negligible. Differences of solidification and dilution phenomenon were also observed between liner welding and spot welding. It was demonstrated the obtained results in this work provided a vital perspective in comprehending the heat and mass transfer phenomenon inside the melt pool during welding and additive manufacturing process.
\end{abstract}

(C) 2020 Elsevier Ltd. All rights reserved.

\section{Introduction}

The interest of welding of dissimilar metals has been increasing in both the scientific research and industrial manufacturing for the advantages on design flexibility, cost control and so on [15]. For example, the joint of 304 stainless steel and nickel, which is widely used in aerospace, high pressure containers and petrochemical industry, has been investigated by former research studies [6,7]. However, defects such as the formation of intermetallic brittle phase and improperly mixed alloy elements caused by the differences in thermal physical and mechanical properties, are still serious challenges in fusion welding of dissimilar metals, which are all mainly determined by the heat and mass transportation inside the WP [8-10]. Consequently, numerical method along with experimental observation is necessary to be carried out to com-

\footnotetext{
* Corresponding authors at: Key Laboratory of Mechanics in Advanced Manufacturing, Institute of Mechanics, Chinese Academy of Sciences, Beijing 100190, China.

E-mail addresses: gyu@imech.ac.cn (G. Yu), xlhe@imech.ac.cn (X. He).
}

prehend the heat transfer, fluid flow and concentration dilution during dissimilar welding process to optimize welding parameters, and hence, improve welding quality.

Heat transfer and fluid flow are still the subject for many studies in welding and additive manufacturing area, and fundamental progress has also been made during the past decades [11-14]. He at al [15] studied the driving mechanism and solidification behavior during laser spot welding of 304SS, and they concluded that the fluid flow inside the melt pool is mainly driven by surface tension. The temperature gradient and solidification rate for laser welding are much larger than that for GTA welding of 304SS. A finite element model was introduced by Traidia et al. [16] to characterize the coupling mechanism between arc and dynamic flow during GTA welding of 304SS, and it was demonstrated that Marangoni convection plays an vital role on dynamic behavior inside the melt pool. Bahrami et al. [17] established a numerical model to investigate the fluid flow for GTA welding of dissimilar metals, and they found that the surface shear stress, which is the main driving force for the Marangoni convection, is mainly affected by the tempera- 


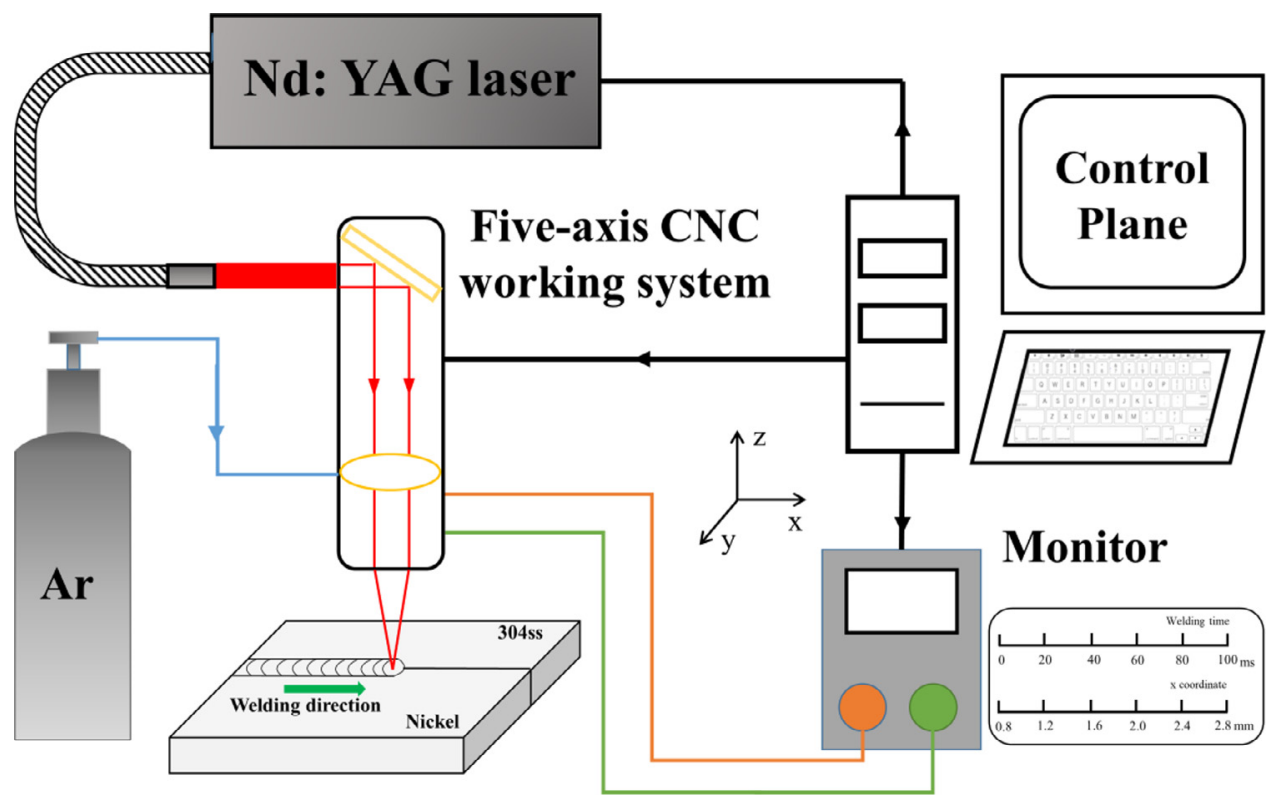

Fig. 1. Schematic diagram for the laser welding system.

ture gradient once the melt pool reaches steady condition. Li et al. [18] proposed a fluid flow model to study the solidification parameter (e.g. temperature gradient $\mathrm{G}$ and solidification growth rate $\mathrm{R}$ ) distribution under different heat input for laser dissimilar welding, and it was observed that the cooling rate (GR) is more prone to being affected by welding heat input compared with morphology parameter(G/R). The peer-reviewed heat transfer and fluid flow studies provide a fundamental comprehension to understand the thermal behavior and flow characteristics, however, the fluid flow characteristics and its correlation with dilution inside the melt pool is still not analyzed in detail. The 3D distribution of solidification parameter at the solidification interface and its effect on solidification phenomenon is also urge to be studied for a deep understanding of the solidification process.

Coupling the mass transportation with fluid flow inside the melt pool is one of the great challenges for dissimilar welding models $[19,20]$. However, alloy element transfer is necessary to be accurately and deeply investigated for the reason that it is closely related to the solidified microstructure and mechanical properties [21,22]. Multicomponent mass transfer in direct laser deposition was investigated by Gan et al. [23] based on an improved 3D numerical model, and it was found that multicomponent diffusion has a non-negligible effect at initial stage. A finite element model was introduced by Bahrami et al. [24] to predict the mass transport in autogenous GTA welding of dissimilar metals, and the velocity field was considered as the decisive factor for the distribution of the alloy elements. For laser spot welding of dissimilar metals, $\mathrm{Hu}$ et al. [9] developed a heat and mass transfer model to simulate the element distribution in laser spot welding, and the speed for mass transport was found the highest at initial stage and decreased with time. Though basic progress has been made in the area of mass transfer, previous work was most focused on explaining the transfer mechanism, especially the effect of diffusion and convection on dilution, and the correlation between dynamic behavior and concentration dilution inside the melt pool has not been studied sufficiently. Besides, different from the time dependent model for spot welding, the continuous melting-solidification process and fast moving speed of heat source results in absolutely nonsymmetric WP and much shorter mixing time in laser linear welding, and require the governing equations solved at the moving coordinate considering a constant scanning speed, which makes the de- velopment of numerical model for linear laser welding of dissimilar metals quite different from that for spot welding and has not been investigated by former studies. In addition, laser linear welding is much more widely used in industrial fabrication [25-27], and the effects of scanning speed, TCST and dynamic viscosity on solute transfer during laser linear dissimilar welding process have not been paid enough attention, which are proved non-negligible factors for mass transfer in this paper.

In this study, an improved three-dimensional model was developed to study the temperature distribution, solidification characteristics, fluid flow and concentration dilution during laser linear welding of SS and Ni. The thermal behavior, including the solidification phenomenon, was firstly analyzed for its basic effect on heat and mass transfer, and then a 3D view study was presented to deeply understand the solidification parameter distribution and solidification phenomenon. The fluid flow characteristics were then investigated in detail for its dominant role in mass transfer $[9,10]$. Next, the correlation between dynamics behavior and concentration dilution was studied using simulation and dimensional analysis methods. Besides, the effects of welding speed, TCST and dynamic viscosity on solute dilution were simulated and compared, and the non-negligible influence of these factors on mass transfer was demonstrated and discussed. Finally, the heat and mass transfer process of laser linear welding was detailedly compared with that of laser spot welding.

\section{Experimental procedure}

As shown in Fig. 1, nickel and 304 stainless steel sheets with size of $60 \mathrm{~mm} \times 40 \mathrm{~mm} \times 2 \mathrm{~mm}$ were welded on a self-developed laser manufacturing system including Nd: YAG continuous fiber laser, five-axis CNC working system and clamping platform. The variation of the position of laser beam center with welding time is also illustrated in Fig. 1. The surface impurities, such as the oil, were removed by acetone before the welding experiments. The compositions of SS and Ni could be found in [6], and not listed here. The welding parameters used in the experiment were shown in Table 1. Then, metallographic samples were obtained through electrical discharge wire cutting machine. After rough grinding, fine grinding, followed by polishing, the samples were etched by $\mathrm{HCL}: \mathrm{HNO}_{3}$ solution with a volume ratio of $3: 1$. To validate the nu- 
Table 1

Welding parameters used in experiments.

\begin{tabular}{llll}
\hline Laser spot & Shielding gas & Laser power & Welding speed \\
\hline $0.57 \mathrm{~mm}$ & Pure $\operatorname{argon}(15 \mathrm{~L} / \mathrm{min})$ & $800 \mathrm{~W}$ & $20 \mathrm{~mm} / \mathrm{s}$ \\
\hline
\end{tabular}

merical predictions of the solidified microstructure and mixed concentration, the treated specimens were then observed and measured on ZEISS EV18 scanning electron microscopy (SEM) equipped with Oxford INCA energy dispersive spectrometer (EDS).

\section{Numerical implement}

In this work, an improved three-dimensional numerical model was developed to study the thermal behavior, fluid flow and mass transfer phenomenon during laser linear welding of 304SS and Ni. The thermal physical properties and parameters used in the simulation are listed in Tables 2 and 3, respectively. To simplify the simulation, the model is formulated under some basic assumptions:

- Fluid flow inside the melt pool is Newtonian, laminar and incompressible; Boussinesq approximation is involved to study the buoyance driven flow inside the melt pool due to the density differences related to temperature variation.

- The laser heat source irritated on the surfaces of the substrates is assumed to be Gaussian distribution, and the effective absorption coefficient is considered as the function of wavelength and the substrate resistivity [18].

- The mushy zone, where the temperature is between the solidus and liquidus, is assumed to be a porous medium with isotropic permeability $[28,29]$.

- Thermal resistance between SS and $\mathrm{Ni}$ is not taken into account.

- The relevant thermal physical properties of the substrate, such as thermal conductivity and dynamic viscosity, are temperature independent.

- The top surface of the melt pool is assumed to be flat, and the vaporization of alloy elements is negligible.

\subsection{Flow model}

The heat transfer and fluid flow in linear welding process is assumed in a steady state with a moving coordinate attached to the laser beam. Based on the assumptions mentioned above, the equations of conservation of mass and momentum are described as:

$\frac{\partial \rho}{\partial t}+\nabla \cdot(\rho \mathbf{u})=0$

$\rho \frac{\partial \boldsymbol{u}}{\partial t}+\rho(\boldsymbol{u} \cdot \nabla) \boldsymbol{u}=\nabla \cdot\left[-p \boldsymbol{I}+\mu\left(\nabla \boldsymbol{u}+(\nabla \boldsymbol{u})^{T}\right)-\frac{2}{3} \boldsymbol{u}(\nabla \cdot \boldsymbol{u}) \boldsymbol{I}\right]$

$$
+\boldsymbol{F}_{\boldsymbol{d}}+\boldsymbol{F}_{\boldsymbol{b}}
$$

Table 3

Parameters used in calculation [9].

\begin{tabular}{ll}
\hline Parameter & Value \\
\hline Power distribution factor & 2 \\
Laser absorption efficiency & 0.26 \\
Ambient temperature & $300 \mathrm{~K}$ \\
Convective heat transfer coefficient at top surface & $100\left(\mathrm{~W} / \mathrm{m}^{2} \mathrm{~K}\right)$ \\
Emissivity & 0.2 \\
Stefan-Boltzmann constant & $5.67 \times 10^{-8}\left(\mathrm{~W} / \mathrm{m}^{2} \mathrm{~K}^{4}\right)$ \\
\hline
\end{tabular}

Where $\rho$ and $\boldsymbol{u}$ denote the density of liquid metal and velocity vector inside the melt pool, respectively. $p$ is pressure of the fluid and $\mu$ represents the viscosity of the liquid mixture.

Source term $\boldsymbol{F}_{\boldsymbol{d}}$ is used to describe the fluid flow in the mushy zone based on Carmen-Kozeny technique [25,26]. Its mathematical description is given as:

$\boldsymbol{F}_{\boldsymbol{d}}=-K_{0} \frac{\left(1-f_{l}\right)^{2}}{\left(f_{l}^{3}+M\right)} \boldsymbol{u}$

In Eq. (3), $K_{0}$ is a parameter associated with the mushy zone morphology, and $M$ is a small positive number used to avoid the division by zero, which are taken as $10^{7}$ and $10^{-4}$ in this work, respectively. $f_{l}$ is the liquid fraction, which could be expressed as:

$$
\begin{array}{cc}
f_{l}=\{0, & 0<T<T_{s} \\
\frac{T-T_{S}}{T_{l}-T_{s}}, & T_{s}<T<T_{l} \\
1, & T>T_{l}
\end{array}
$$

where $T_{S}$ and $T_{S}$ are the alloy solidus and liquidus temperature, respectively.

Another source term in Eq. (2) is $\boldsymbol{F}_{\boldsymbol{b}}$, and it consists of the body force generated by the temperature and concentration related gravity-driven buoyance force, viz.,

$\boldsymbol{F}_{\boldsymbol{b}}=-\rho\left[\beta_{T}\left(T-T_{r e f}\right)+\beta_{c}\left(C-C_{r e f}\right)\right] \mathbf{g}$

In this equation, $\beta_{T}$ and $\beta_{c}$ denote the temperature volumetric expansion coefficient [7] and composition volumetric expansion coefficient [17], respectively. $T_{\text {ref }}$ is the reference temperature ( $300 \mathrm{~K}$ ) and $T$ is the local temperature inside the melt pool. In addition, the properties $C$ and $C_{r e f}$ represent the local and reference concentration, respectively. The vector $\mathbf{g}$ is the gravitational acceleration acting on the negative $\mathrm{z}$ direction.

\subsection{Temperature field}

The equation of energy is given as:

$$
\rho C_{p} \frac{\partial T}{\partial t}+\rho C_{p} \boldsymbol{u} \cdot \nabla T=\nabla \cdot(K \nabla T)-\rho \frac{\partial(\Delta H)}{\partial t}-\rho \boldsymbol{u} \nabla(\Delta H)
$$

Table 2

Thermal physical properties used for the simulation $[6,17]$.

\begin{tabular}{lll}
\hline Property & $304 \mathrm{SS}$ & $\mathrm{Ni}$ \\
\hline Liquidus temperature $(\mathrm{K})$ & 1727 & 1735 \\
Solidus temperature $(\mathrm{K})$ & 1672 & 1730 \\
Heat of fusion $(\mathrm{kJ} / \mathrm{kg})$ & 272 & 290 \\
Specific heat of solid $(\mathrm{J} / \mathrm{kg} \bullet \mathrm{K})$ & 711.28 & 515 \\
Specific heat of liquid $(\mathrm{J} / \mathrm{kg} \bullet \mathrm{K})$ & 836.8 & 595 \\
Thermal conductivity of solid $(\mathrm{W} / \mathrm{m} \bullet \mathrm{K})$ & 19.2 & 60.7 \\
Thermal conductivity of $\mathrm{liquid}(\mathrm{W} / \mathrm{m} \bullet \mathrm{K})$ & 50 & 150 \\
Density of solid metal $\left(\mathrm{kg} / \mathrm{m}^{3}\right)$ & 7450 & 8900 \\
Density of liquid metal $\left(\mathrm{kg} / \mathrm{m}^{3}\right)$ & 6910 & 8880 \\
Dynamic viscosity $(\mathrm{kg} / \mathrm{m} \bullet \mathrm{s})$ & $6.70 \times 10^{-3}$ & $3.68 \times 10^{-3}$ \\
Surface tension $(\mathrm{N} / \mathrm{m})$ & 1.872 & 1.778 \\
Temperature coefficient of surface tension $(\mathrm{N} / \mathrm{m} \bullet \mathrm{K})$ & $-4.30 \times 10^{-4}$ & $-3.40 \times 10^{-4}$ \\
Liquid volume thermal expansion $\left(\mathrm{K}^{-1}\right)$ & $1.96 \times 10^{-5}$ & $4.50 \times 10^{-5}$ \\
Liquid volume concentration expansion & 0.078 & 0.078 \\
Effective mass diffusivity $\left(\mathrm{m}^{2} / \mathrm{s}\right)$ & $7 \times 10^{-7}$ & $7 \times 10^{-7}$ \\
\hline
\end{tabular}


Where $C_{p}$ is the specific heat, $K$ is the thermal conductivity, and $\Delta H$ is the latent enthalpy change caused by the phase change in the computational cell, which is described as:

$\Delta H=L f_{l}$

where $L$ denotes the latent heat of fusion. It should be noted that the latent heat, which is also known as the enthalpy of fusion, has nonnegligible effect on the heat and mass transfer process and thus considered in the equation of energy.

In this study, to illustrate the heat and mass transfer phenomenon, all the governing equations are solved based on the Cartesian coordinate attached to the moving laser heat source. Actually, the velocity field obtained from the Navi-Stokes equations in the manuscript is the fluid velocity relative to the moving coordinate, which moves at a constant speed same as the welding speed. The welding speed $\boldsymbol{u}_{w}$ is $20 \mathrm{~mm} / \mathrm{s}$ and along with the positive Xaxis direction. The fluid velocity under fixed Cartesian coordinate is described as:

$\overline{\boldsymbol{u}}=\boldsymbol{u}-\boldsymbol{u}_{\boldsymbol{w}}$

Where $\boldsymbol{u}_{\boldsymbol{w}}=u_{w} i$, and $i$ is the unit vector along the $\mathrm{x}$ direction. As a result, the next relations could be obtained.

$\nabla \overline{\boldsymbol{u}}=\nabla \boldsymbol{u}$

$\left(\overline{\boldsymbol{u}}+\boldsymbol{u}_{w}\right) \cdot \nabla \overline{\boldsymbol{u}}=\boldsymbol{u} \cdot \nabla \boldsymbol{u}$

$\nabla^{2} \overline{\boldsymbol{u}}=\nabla^{2} \boldsymbol{u}$

Consequently, Eqs. (1), (2) and (6) could be written as follows:

$\frac{\partial \rho}{\partial t}+\nabla \cdot(\rho \overline{\boldsymbol{u}})=0$

$\rho \frac{\partial \overline{\boldsymbol{u}}}{\partial t}+\rho(\overline{\boldsymbol{u}} \cdot \nabla) \overline{\boldsymbol{u}}=\nabla \cdot\left[-p \boldsymbol{I}+\mu\left(\nabla \overline{\boldsymbol{u}}+(\nabla \overline{\boldsymbol{u}})^{T}\right)-\frac{2}{3} \overline{\boldsymbol{u}}(\nabla \cdot \overline{\boldsymbol{u}}) \boldsymbol{I}\right]$ $+\boldsymbol{F}_{\boldsymbol{d}}+\boldsymbol{F}_{\boldsymbol{b}}-\rho \boldsymbol{u}_{\boldsymbol{w}} \cdot \nabla \overline{\boldsymbol{u}}$

$\rho C_{p} \frac{\partial T}{\partial t}+\rho C_{p} \overline{\boldsymbol{u}} \cdot \nabla T=\nabla \cdot(K \nabla T)-\rho \frac{\partial(\Delta H)}{\partial t}-\rho \overline{\boldsymbol{u}} \nabla(\Delta H)$ $-\rho C_{p} \boldsymbol{u}_{\boldsymbol{w}} \cdot \nabla T-\rho \boldsymbol{u}_{\boldsymbol{w}} \nabla(\Delta H)$

\subsection{Concentration field}

To model the mass transfer inside the melt pool during dissimilar welding, the effects of convection and diffusion are considered. The transport equation for certain species $C_{i}$ could be described as:

$\frac{\partial C_{i}}{\partial t}+\left(\boldsymbol{u}_{\boldsymbol{w}}+\overline{\boldsymbol{u}}\right) \cdot \nabla C_{i}=\nabla \cdot\left(D_{i} \nabla C_{i}\right)+S$

In Eq. (15), $C_{i}$ and $D_{i}$ represent the local concentration and diffusion coefficient of certain alloy element inside the melt pool. The source term $S$ is assumed to be 0 in this study because there are no material addition and chemical reaction during the laser dissimilar welding process.

\subsection{Boundary conditions}

The heat boundary at top surface is given as:

$-K \frac{\partial T}{\partial z}=\frac{2 Q_{e}}{\pi r_{b}{ }^{2}} \exp \left(-\frac{2 r^{2}}{r_{b}^{2}}\right)-h_{c}\left(T-T_{r e f}\right)-\sigma_{b} \varepsilon\left(T^{4}-T_{r e f}^{4}\right)$

In Eq. (16), the first term at right hand is the energy input caused by laser heat source, and $Q_{e}$ is the absorbed laser power associated with laser heat input and absorption efficiency of the substrates. The properties $r$ and $r_{b}$ represent the distance to the laser center and the effective laser radius, respectively. Besides, the second term and third term are the heat loss caused by heat convection and radiation, respectively. $h_{c}$ represents the heat transfer coefficient of convection. $\sigma_{b}$ denotes the Stefan-Boltzmann constant, and $\varepsilon$ is the emissivity. The heat boundary condition for other sides of the substrates only consists of the second and third term of Eq. (15) because there is no heat input on these sides.

The gas/liquid interface is assumed to be flat, and the effect of free surface deformation on heat and momentum boundaries is neglected. Consequently, the boundary condition of momentum equation at top surface could be expressed as:

$\mu \frac{\partial u}{\partial z}=f_{l} \frac{d \gamma}{d T} \frac{\partial T}{\partial x}+f_{l} \frac{d \gamma}{d C} \frac{\partial C}{\partial x}$

$\mu \frac{\partial v}{\partial z}=f_{l} \frac{d \gamma}{d T} \frac{\partial T}{\partial y}+f_{l} \frac{d \gamma}{d C} \frac{\partial C}{\partial y}$

In Eqs. (17) and (18), the two terms at right hand represent the surface shear stress caused by temperature and concentration variations, respectively, which act as the momentum boundary on the top surface. $\gamma$ denotes the surface tension of liquid metal. It should be noted that the concentration derivative term is only comparable with the temperature derivative term at initial stage, and concentration effect is negligible at steady condition [17].

There is no mass addition for all the boundaries of the substrate, consequently, the species dilution boundary condition could be described as:

$\left(-D_{c_{i}} \nabla C_{i}+C_{i} \boldsymbol{u}_{\boldsymbol{c}_{\boldsymbol{i}}}\right) \cdot \mathbf{n}=0$

In Eq. (19), the first and second term in the parentheses denote the diffusive and convective species fluxes, respectively. $D_{c_{i}}$ and $\boldsymbol{u}_{\boldsymbol{c}_{\boldsymbol{i}}}$ here denote the coefficient of diffusivity and velocity of the mass flux, respectively. Additionally, the initial value of concentration is set as uniform distribution at each material and smoothed at the interface of two substrates.

\subsection{Material properties}

The material properties of the solution are assumed temperature independent, and the used material properties for the model are listed in Table 2. To smooth the differences of thermal physical properties between SS and $\mathrm{Ni}$, a Heaviside function with smooth method is used in the transition. For example, the transitions of density and specific heat in liquid phase are illustrated in Fig. 2, where the transition is smoothed near the interface of SS and Ni.

The thermal physical properties, such as heat capacity and thermal conductivity, are defined as the function of mass fraction and liquid volume fraction in the mushy zone, where the domain consists of liquid and solid mixture. The properties of mixture are defined as:

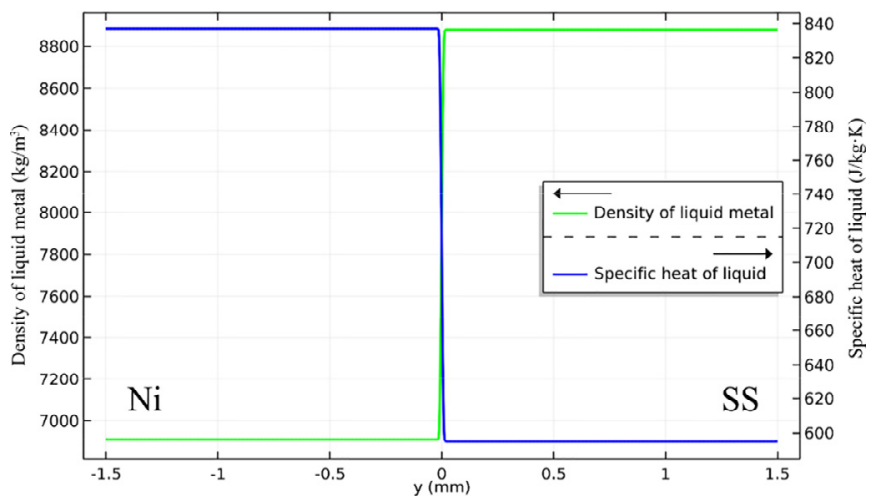

Fig. 2. Transitions of material density and specific heat in liquid phase. 

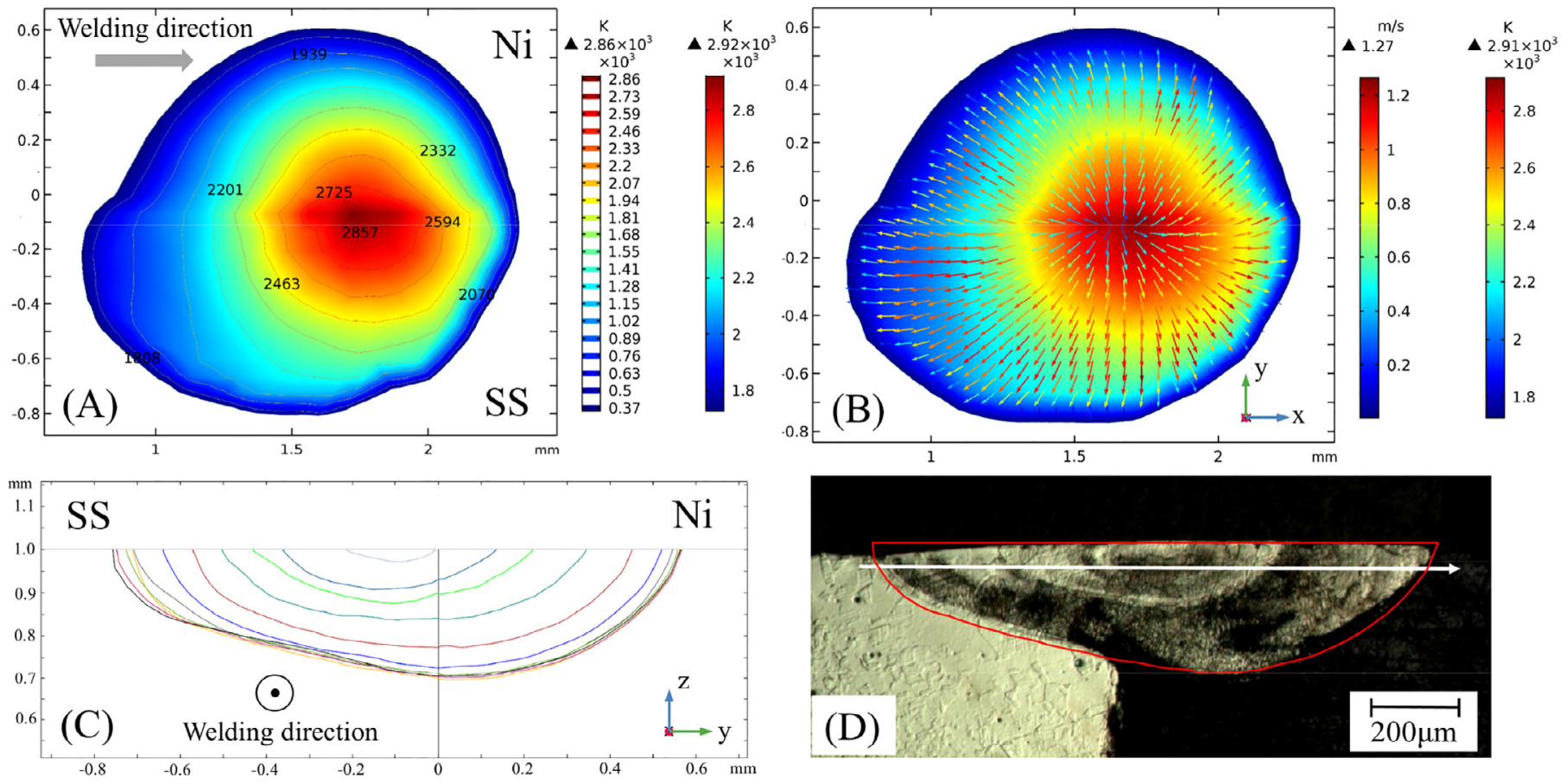

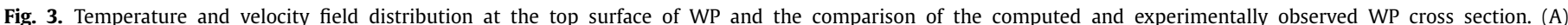

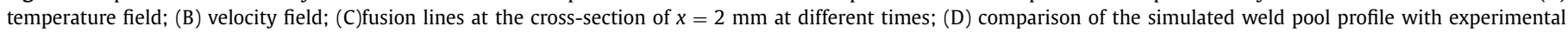
result [6].

$C_{p}=f_{s} C_{p s}+f_{l} C_{p l}$

$\rho=f_{s} \rho_{s}+f_{l} \rho_{l}$

$K=\left(g_{s} / K_{s}+g_{l} / K_{l}\right)^{-1}$

$D=f_{l} D_{l}$

where $f_{l}$ and $g_{l}$ are the mass fraction and volume fraction of the liquid phase, respectively. The subscripts $s$ represents the solid phase, and $l$ denotes the liquid phase in this study.

Quadratic polynomials were used for the discretization for the whole domain and all the equations were solved using PARDISO solver. The mesh grids consist of 200,114 domain units, and a maximum time step of $0.1 \mathrm{~ms}$ was employed. To exclude the meshsensitivity, mesh convergency test has been carried out firstly by varying the maximum mesh size.

\section{Results and discussion}

\subsection{Thermal behavior}

\subsubsection{Heat transfer}

The temperature and velocity distribution at the top surface of the WP when the WP reaches steady condition are depicted in Fig. $3 \mathrm{~A}$ and $\mathrm{B}$, respectively. Resulting from the difference of thermal physical properties between $\mathrm{SS}$ and $\mathrm{Ni}$, a nonsymmetrical melt area is observed, and it is larger at SS side at top surface. Besides, the melted width at top surface is also larger at SS side (see Fig. 3A), however, the melted depth at cross section is larger at Ni side (see Fig. 3C).The peak temperature is around $2900 \mathrm{~K}$, and the temperature isotherms are obviously denser at the front area, which means that the temperature gradient is much higher here. A centrally outward fluid flow mainly driven by surface tension is also found, and the velocity increases from the center to the periphery as a re- sult of negative surface tension temperature coefficient [21]. Under the effect of intense Marangoni convection, the maximum velocity reaches $1.27 \mathrm{~m} / \mathrm{s}$, and this convection plays an important role in the heat transfer and concentration dilution during laser welding of miscible dissimilar metals. To obtain the final simulated weld pool profile, liquidus isotherms at the cross-section of $x=2 \mathrm{~mm}$ at different times (from the time weld pool reaches $x=2 \mathrm{~mm}$ to the time it absolutely leaves $x=2 \mathrm{~mm}$ ) represented by different colors are plotted together in Fig. 3C. The final melted area includes all the materials in the substrate that have experienced the melting-solidification process, thus the final fusion line could be obtained by superposition of the fusion lines at different times. To validate the numerical model, which is next used to study the solidification characteristics and mass transfer, the final fusion line obtained from the superposition of fusion lines at different times is plotted in Fig. 3D and compared with the experimental result on weld width, weld depth and solid/liquid boundary (fusion line), and the simulated profile shows good correspondence with the experimental result.

\subsubsection{Solidification characteristics}

According to the mechanism of welding metallurgy [30,31], the final solidified microstructure of the melt pool is closely related to the transient thermal behavior and could be predicted by the solidification parameters including temperature gradient $\mathrm{G}$, solidification rate $R$, and their combined parameters $G / R$ and $G R$. The morphology parameter $G / R$ is related to the microstructure morphology, while the cooling rate GR determines the size of the grains. The larger the cooling rate GR, the finer the microstructure, and the transition from cellular dendrites to columnar dendrites to equiaxed dendrites is prone to occurring with the decrease of $\mathrm{G} / \mathrm{R}$. Actually, the crystals grow across the whole WP and solidification phenomenon is related to the averaged/homogenized solidification parameters, not the dynamic values. Thanks to the assumptions that solidification could be assumed steady when the WP reached steady stage and the values of solidification parame- 
ters for one point at the cross section could be represented by the values for one point at the solid/liquid interface at steady stage, which have been investigated in former studies $[3,23,32]$, the solidification parameters obtained from the transient thermal analysis could be used to study the solidification phenomenon at steady condition. For example, the value of GR for one point at the interface is equal to that for another point at the solidification interface at another welding time that have the same y coordinate and $\mathrm{z}$ coordinate. To deeply study the solidification phenomenon, the distribution of $G / R$ and $G R$ at the solid/liquid interface are plotted in Fig. 4 with color contour from $\mathrm{x}$-axis view, $\mathrm{y}$-axis view, $\mathrm{z}$-axis view and $3 \mathrm{D}$ view, respectively, and the distribution of $\mathrm{G}$ and $\mathrm{R}$ are not discussed for the aim of simplicity. Compared with the computed 2D solidification parameters in former study [3,23,32], this 3D distribution presented in this study provides an intuitive observation of the solidification parameter distribution at different locations. In addition, the variation of solidification characteristics along the welding direction (x-axis) and the solidification interface (see the color map in Fig. 4), where the solidification occurs, are also depicted in Fig. 4. Moreover, as a result of the numerical oscillation near the mushy zone, the $2 \mathrm{D}$ plots of $G R$ and $G / R$ are prone to showing noisy results around the mushy zone in simulation study [32], which could be avoided in the 3D study.

As shown in Fig. 4A, the cooling rate GR decreases along both the depth direction (negative $\mathrm{z}$-axis) and the welding direction ( $\mathrm{x}$ axis). The maximum and minimum GR are observed at the top area and bottom of the WP, respectively, which is $5.02 \times 10^{4} \mathrm{~K} / \mathrm{s}$ and $1.28 \times 10^{3} \mathrm{~K} / \mathrm{s}$. Besides, the GR at SS side is found much larger than that at $\mathrm{Ni}$ side. According to above analysis of cooling rate, a finer solidified structure is expected to be observed at the top part and located at SS side. As illustrated in Fig. 4B, there is an obvious increase of $\mathrm{G} / \mathrm{R}$ along the depth direction and the welding direction. The peak value is $70 \mathrm{~K} \bullet \mathrm{s} / \mathrm{mm}^{2}$ and $3.83 \times 10^{7} \mathrm{~K} \bullet \mathrm{s} / \mathrm{mm}^{2}$, which are found near the bottom and top part, respectively. However, there is no obvious difference of $G / R$ between SS side and $\mathrm{Ni}$ side, which means that the morphology of the solidified WP at two sides maybe similar. In conclusion, the change of morphology from planar, cellular dendrites, columnar dendrites to equiaxed dendrites is prone to occurring along the z-axis direction at SS side.

To validate the numerical predication of the solidified microstructure, SEM has been carried out. The WP for dissimilar welding is the mixture of different materials, and symbol $\mathrm{Ni}$ and symbol SS in the subfigures of Fig. 5 represent the solidified liquid mixture at nickel side and 304SS side, respectively. As depicted in Fig. 5, the transition from planar to coarser cellular to equiaxed dendrites is observed with the increase of cooling rate GR and the decrease of morphology parameter $\mathrm{G} / \mathrm{R}$ at SS side. Besides, resulting from higher cooling rate, the scale of the microstructure at SS side is finer than that at $\mathrm{Ni}$ side.

\subsection{Fluid flow}

To investigate the flow characteristics inside the WP, which is closely correlated to the concentration dilution, the velocity field on cross sections along $\mathrm{x}$-axis, $\mathrm{y}$-axis and $\mathrm{z}$-axis are numerically studied, respectively. As presented from Figs. 6 to 8, the magnitude is shown by color contour and its direction is depicted by white arrows. The initial location of heat source is $x=0.8 \mathrm{~mm}$ and the welding speed is $20 \mathrm{~mm} / \mathrm{s}$ in this simulation, thus the position of the center of laser beam is exactly at $x=1.7 \mathrm{~mm}$ at this moment with the welding time of $45 \mathrm{~ms}$. Larger velocity is found near the gas/liquid surface, which indicates that the Marangoni convection is more intense here. Additionally, the fluid flow field on three yz-cross sections, shown in Fig. $6 \mathrm{~A}$, are depicted from Fig. $6 \mathrm{~B}$ to D. At plane 1, as shown in Fig. 6B, liquid metal flow is generally from SS side to Ni side, which contributes to the alloy element mixing in the WP, such as the $\mathrm{Cr}$ and Fe at SS substrate. However, as shown in Fig. $3 \mathrm{C}$ and D, part of the flow is generated from $\mathrm{Ni}$ side to SS side and this trend becomes weaker from plane2 to plane3. This phenomenon could be explained by the difference of physical properties between SS and $\mathrm{Ni}$. At initial stage (plane1), the dynamic viscosity at SS side is much larger than that at $\mathrm{Ni}$ side, and fluid is prone to flowing towards Ni side, where it is less restricted. With the WP evolution, this difference of viscosity becomes smaller due to the dilution inside the WP, but the surface tension, which is the mainly driving force of the fluid flow, is still larger at SS side, resulting in the flow towards SS side. It should be noted that both the convection and dilution happen in three dimension and should be studied in different directions to understand the heat and mass transfer process accurately.

Velocity distribution on three xz-sections, shown in Fig. 7A, are presented from Fig. 7B to D, respectively. To illustrate the fluid flow clearly, the positions of plane 4 and plane 5 are moved along positive $\mathrm{z}$ axis direction in Fig. 7A. As shown in Fig. 7, compared with plane 4 and plane 6 , the convection at plane5 (the interface of SS and $\mathrm{Ni}$ ) is obviously weaker. This phenomenon indicates that the mixing along $\mathrm{x}$ direction is mainly generated at the position away from the interface of SS and $\mathrm{Ni}(y=0)$, because convection is the main transfer mechanism $[9,10]$ and the vortex is more intense at plane 4 and plane 6 . At Ni side, plane 4 , the fluid flow is generally from front part to the rear side; on the contrast, it is from back to the front at plane 6 , SS side. It should be noted that the concentration dilution inside the WP, which is closely related to the convection flow inside the melt pool, is also a 3D phenomenon and presents special characteristics in different directions, resulting from the complex variations of liquid metal flow.

The temperature and velocity field in $3 \mathrm{D}$ view is plotted in Fig. 8A, and all the fluid flow on cross sections shown from Fig.6 to Fig. 8 are partially of the 3D Marangoni convection shown in Fig. 8A. As illustrated, two xy cross sections are selected to study the flow characteristics in $\mathrm{z}$ direction. The convection is obviously more intense at upper part of the WP. Fluid flow from periphery to center is observed both at plane $7(z=0.8 \mathrm{~mm})$ and plane 8 $(z=0.9 \mathrm{~mm})$, but the flow at plane 8 is stronger, which indicates that element is mainly mixed near the top surface and then transported towards bottom by the $3 \mathrm{D}$ vortex flow. The inside flow is found stronger at both center and periphery than any other areas, which is part of the 3D Marangoni flow (see Fig. 8A). Besides, part of the inside flow at xy plane is along the positive $\mathrm{x}$ direction and this branch flow is much stronger. This $\mathrm{x}$ direction branch flow is of great importance for the dilution phenomenon near the bottom of the melt pool because the Marangoni convection is more intense at upper side.

\subsection{Mass transportation}

\subsubsection{Correlation between flow characteristics and concentration dilution}

Alloy elements transfer during dissimilar welding, such as $\mathrm{Cr}$ and $\mathrm{Ni}$, is dominated by diffusion and convection mechanism. The non-dimensional number, Peclet number for mass transfer, can be used to describe the relative importance of these two different mass transfer mechanisms $[9,23]$, and its expression is:

$P_{e c}=\frac{U L_{R}}{\alpha}$

Where $U$ and $L_{R}$ are the characteristic velocity and length, respectively. The $L_{R}$ in this study is taken as the radius of the WP, which is about $0.6 \mathrm{~mm}$. Based on the physical properties listed in Table 2 and calculated parameters, the $P e_{c}$ in this paper is computed on the order of 1000 , which means that the main transfer 

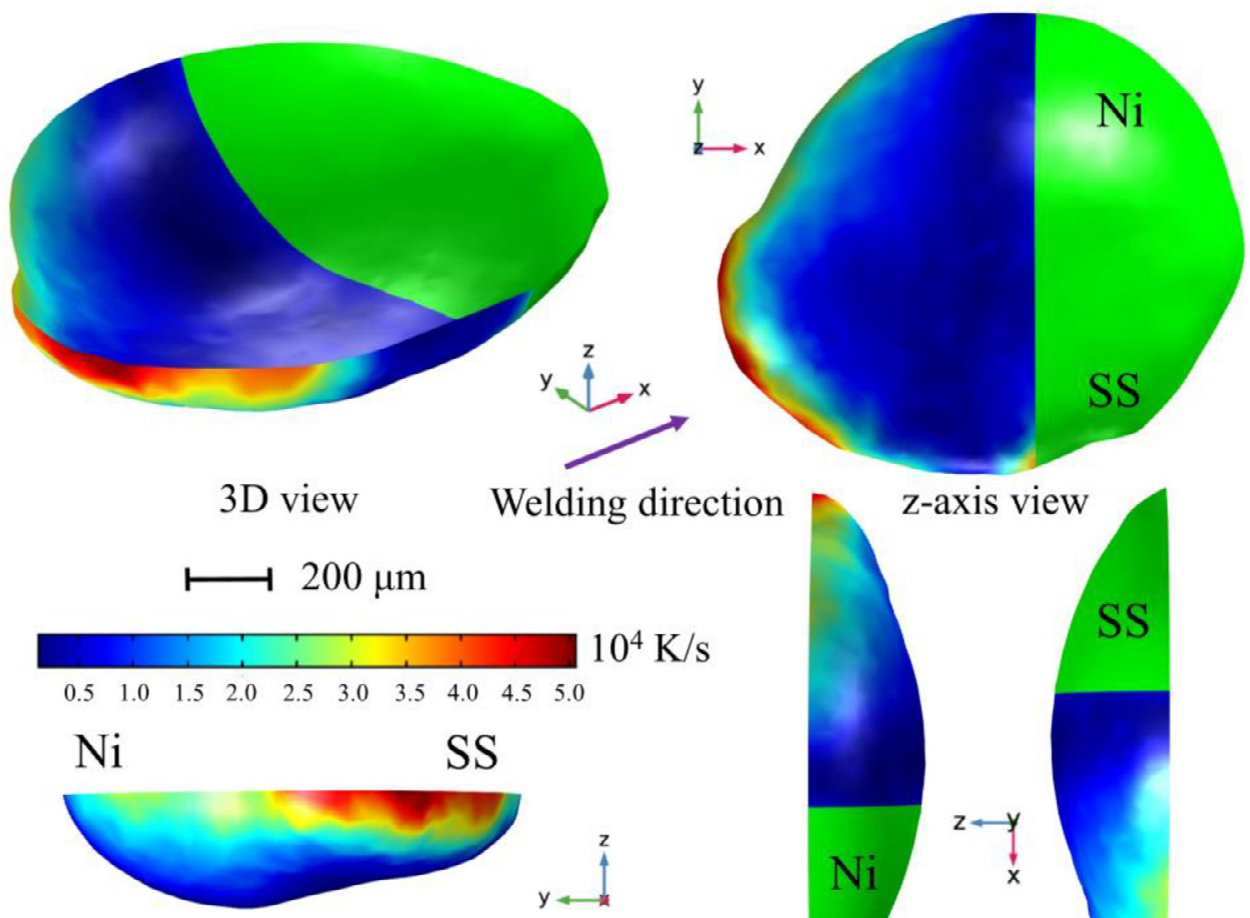

$\mathrm{x}$-axis view

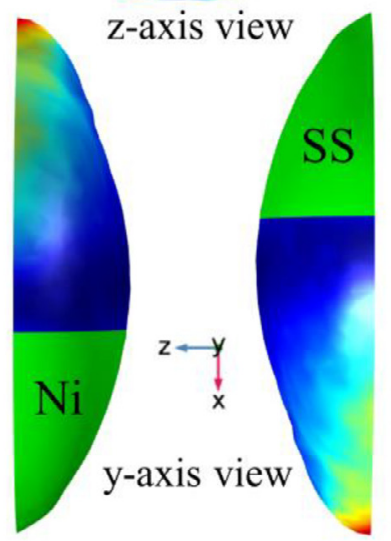

(A) Distribution of cooling rate GR

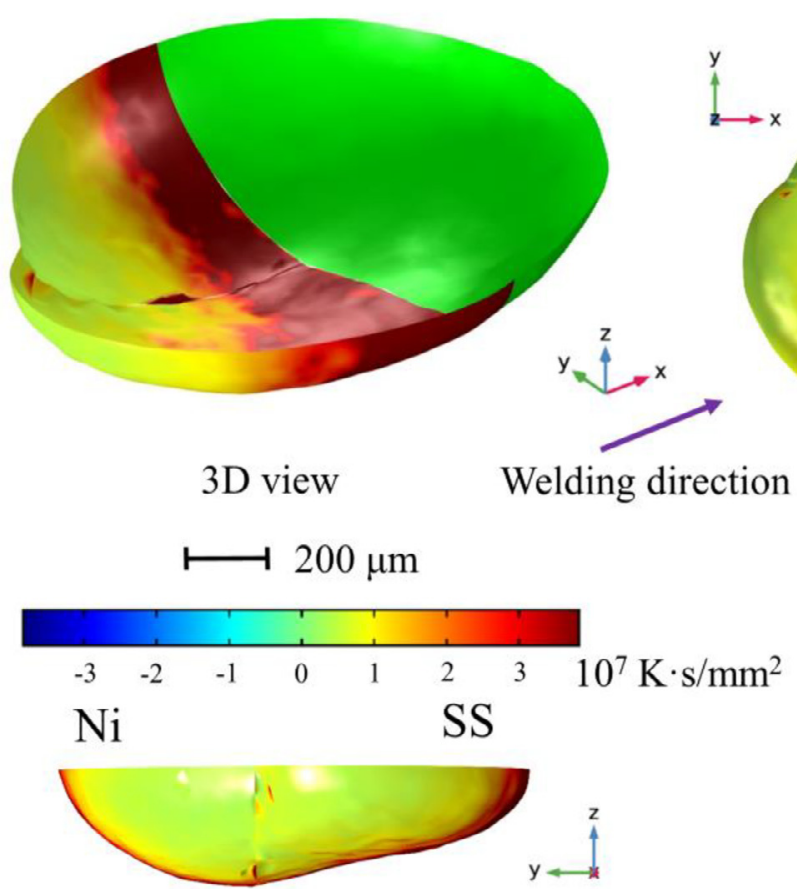

$\mathrm{x}$-axis view
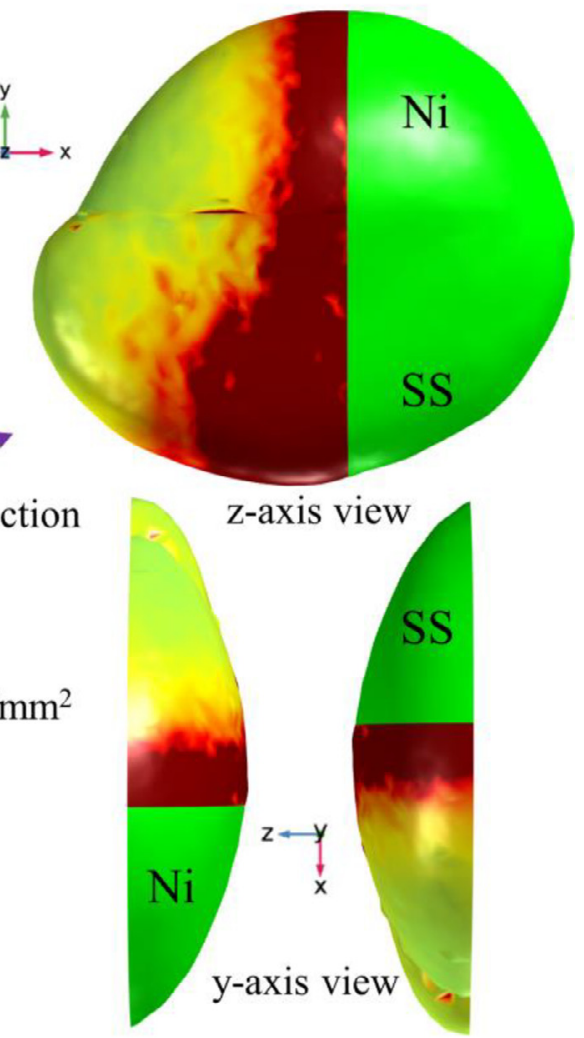

(B) Distribution of morphology parameter $\mathrm{G} / \mathrm{R}$

Fig. 4. Three-dimensional distribution of solidification parameters on the solidification interface. 


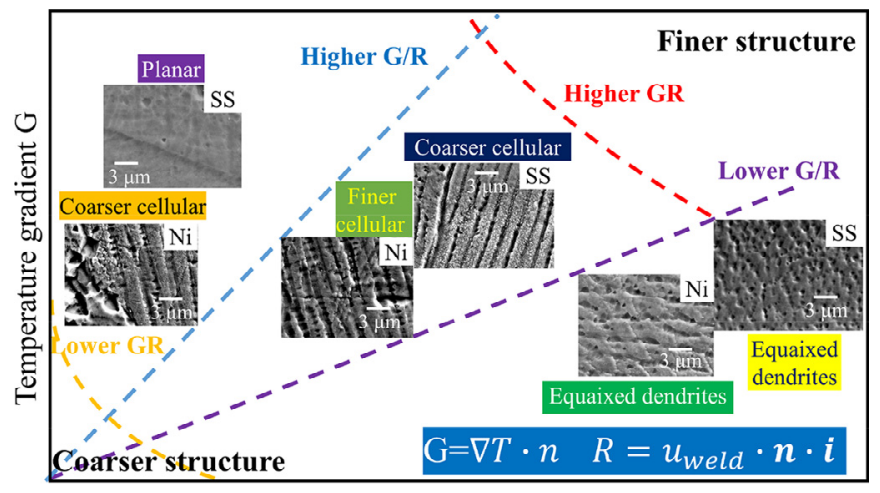

Solidification rate $\mathrm{R}$

Fig. 5. Experimental validation of the simulation prediction based on the calculated solidification parameters.

mechanism is convection and the effect of diffusion could be ignored.

The evolution of $\mathrm{Cr}$ element distribution at top surface of the WP, which is characterized by weight fraction, is depicted in Fig. 9. The weight fraction is shown by color map and the velocity field is plotted by white arrows. Though the $\mathrm{Cr}$ distribution is becoming relatively uniform with the WP evolution, there is still a zone near the fusion line where the velocity is obviously smaller and the mixing is much weaker, which is the so-called unmixed zone (UMZ) [31]. The dynamic viscosity is enormous in UMZ and fluid flow is restricted in this area, hence, it is difficult for $\mathrm{Cr}$ element to be transported here and its weight fraction experiences a great change. As shown in Fig. 9, it takes about $40 \mathrm{~ms}$ for the WP to reach steady condition. The nonuniform area near the boundary decreases with time but it still could be observed through the whole welding process. In addition, as shown from Fig. 6B to Fig. $6 \mathrm{D}$, the velocity is larger at SS side than that at $\mathrm{Ni}$ side and the max velocity is also observed at SS side [6], resulting in more uniform dilution at SS side, which is also illustrated in the analysis of $\mathrm{Cr}$ evolution from Fig. 9A to D

To study the mass transport characteristics inside the WP, dilution phenomenon on different sections is analyzed in detail. It should be noted that the positions of the cross sections from Plane1 to Plane 8 shown in this part are same as that in the part 4.2. Fe concentration on three yz sections, shown in Fig. 10A, are presented from Fig. 10B to D. At plane 1, Fe is mainly transported and mixed in the WP by part of the 3D fluid flow from SS side towards Ni side, and mainly performed at upper part due to Marangoni effect [6]. At the middle part of the WP, plane 2, portion of the flow changes from $\mathrm{Ni}$ side to SS side, and makes $\mathrm{Fe}$ more uniformly distributed at $\mathrm{Ni}$ side. Meanwhile, it could be predicted that Ni will be transported from nickel side to SS side for this branch flow. However, from plane 2 to plane 3, Fe is not obviously transported between SS and Ni substrate, but its concentration at single side becomes more uniform resulting from the two single vortex flow at each side, which is part of the 3D Marangoni convection inside the WP (see Fig. 3B and C). In detail, elements in SS substrate, such as $\mathrm{Cr}$ and $\mathrm{Fe}$, are mainly mixed at the front part of the WP, but Ni is mostly transported at middle part. The redistribution of alloy elements, which makes the elements uniformly mixed at each side, is observed more significant at back.

Three $\mathrm{xz}$ sections are also selected to study the mass transportation phenomenon, and their coordinates along with Fe distribution are illustrated from Fig. 11A to D, respectively. In Fig. 11B, the partially backward flow observed at central area is benefit for the Fe element dilution at Ni side, but the partially frontward flow at SS side, shown in Fig. 11D, makes it difficult for Fe mixing and may lead to a nonuniform distribution in the solidified structure. However, this branch flow at SS side obviously contributes the dilution for $\mathrm{Ni}$, which is mainly transported at middle part of the WP. Consequently, alloy elements from SS side ( $\mathrm{Fe}$ and $\mathrm{Cr}$ ) are mainly transported to Ni side at front part of the WP and then to the back,

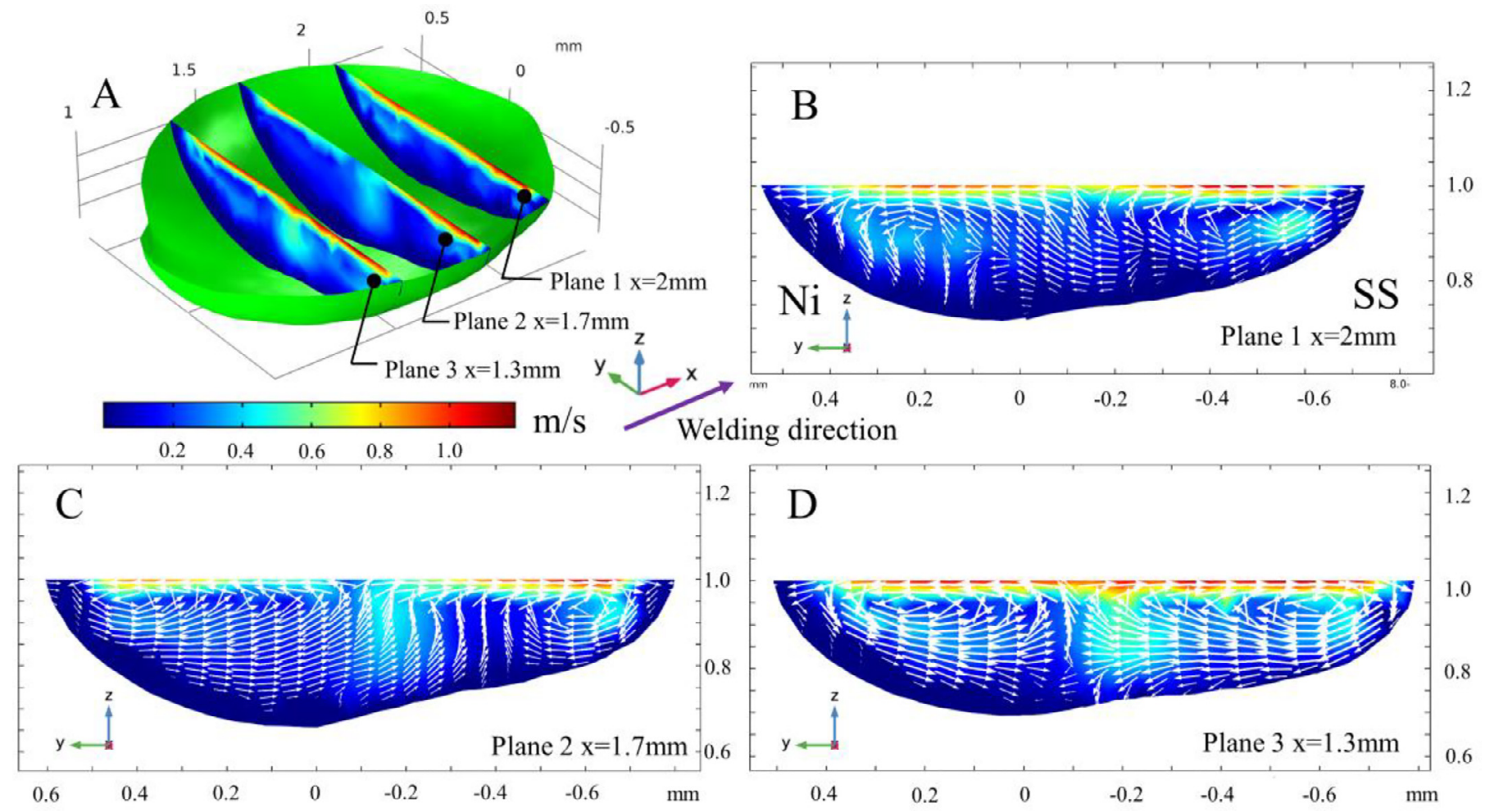

Fig. 6. Fluid flow inside the WP on yz-plane. (A) WP configuration and coordinate of three representative yz-planes;(B) $x=2 \mathrm{~mm}$;(C) $x=1.7 \mathrm{~mm}$; (C) $x=1.3 \mathrm{~mm}$. 


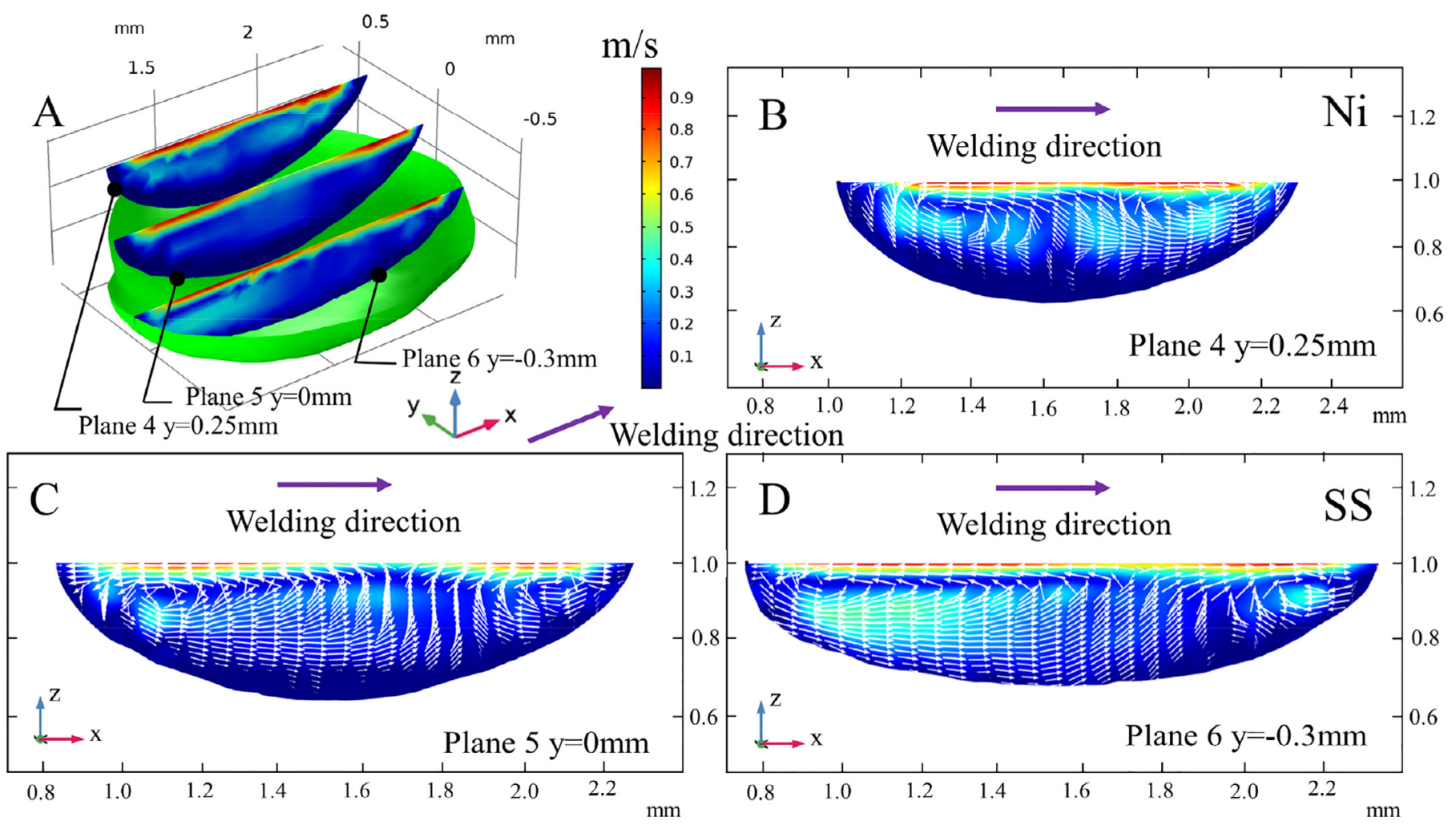

Fig. 7. Fluid flow inside the WP on xz-plane. (A) WP configuration and coordinate of three representative $\mathrm{xz}-\mathrm{planes}$;(B) $y=0.25 \mathrm{~mm}$;(C) $y=0$; (C) $y=-0.3 \mathrm{~mm}$.

but Ni from nickel substrate is mainly mixed at middle part of the WP and then transported to front part and rear side. Besides, the vortex convection, observed from Fig. 11B to D, is benefit for the dilution along $\mathrm{z}$ direction.

As illustrated in Fig. 12, Fe element distribution at xy plane is analyzed on plane $7(z=0.8 \mathrm{~mm})$ and plane $8(z=0.9 \mathrm{~mm})$. A nonuniform distribution of Fe is obviously observed, and its weight fraction is higher at SS side than that at Ni side. Compared with the $\mathrm{Cr}$ distribution at $z=1 \mathrm{~mm}$, shown in Fig. 9, it could be concluded that mass field is more uniform at upper side of the WP as a result of more intense convection at this area (see Fig.8 ). Elements from the periphery are transported to the center by the centrally towards flow and thus concentrated in the central area, and then transported to the front part by this branch flow along $\mathrm{x}$-axis direction. In addition, as shown at plane 7, liquid metal flow near the periphery of $\mathrm{Ni}$ side is stronger than that at SS side, thus the dilution near the bottom area is predicted to be more uniform at Ni side. Finally, mixing is carried out through the intense vortex flow near the welding center line. Transported by the 3D convection flow, elements are then diluted in different directions.

The composition profile of $\mathrm{Ni}, \mathrm{Fe}$ and $\mathrm{Cr}$ is presented in Fig. 13 for the case calculated by the mass transfer model and the case measured by the EDS experiment, respectively. The EDS measurement was carried out at the cross section along a scanning line below the top surface (see Fig. 3D). As depicted in Fig. 13, the simulated alloy element distribution agree well with the measured result.

\subsubsection{Effect of welding speed on concentration dilution}

To investigate the effect of welding speed on mass transfer, numerical studies of velocity field and alloy elements distribution under different welding speed with the laser power of $800 \mathrm{~W}$ are carried out. As illustrated in Fig. 14, the WP dimension and convection flow become smaller and weaker with the increase of welding speed. The enhanced fluid flow in case 1 contributes to the dilu- tion at each side and the mixing in the WP. Based on former study [32], both the $\mathrm{Pe}_{\mathrm{c}}$ and solidification time increase as a result of larger WP dimensions induced by lower welding speed. Therefore, as shown in Fig. 14, a more uniform distribution is induced by lower welding speed as a result of more intense flow and longer mixing time. In addition, the difference of alloy element distribution is not so obvious in the change from case 1 to case 2 as that from case 2 to case 3 . This phenomenon could be explained that the difference of WP dimension between case 1 and case 2 is also much smaller than that from case 2 to case3, resulting in smaller difference in fluid flow and solidification time. Consequently, there may be a range of welding speed, and both the fluid flow and mass transfer change a little within this range (case 1 and case 2 are include) but change a lot beyond this range (case 3 is included). One possible method to control the mass transfer process is to use a higher welding speed but restrict it in a reasonable range.

As mentioned above, mass transfer phenomenon is closely correlated with the joint quality. For example, the formation of unmixed zone (UMZ) and its effect on mechanical properties of the joint are still the challenging problems in dissimilar welding, and this UMZ is usually formed near the fusion boundaries due to the nonuniform mass transfer. The intermetallic brittle phase is prone to occurring in this area, which could seriously lower the welding quality [31]. A more uniformly mixed WP induced by more intense fluid flow and larger WP dimension could also restrict the possible formation of UMZ and improve its mechanical properties [32]. Consequently, the speed of laser linear welding should be restricted within a reasonable range to avoid the generation of UMZ though higher efficiency is one of the main advantages of laser welding.

\subsubsection{Correlation between dynamic properties and concentration dilution}

Based on former studies [21,30], surface tension is the function of temperature and concentration of surface-active elements (e.g. oxygen and sulfur) and the temperature coefficient of surface ten- 

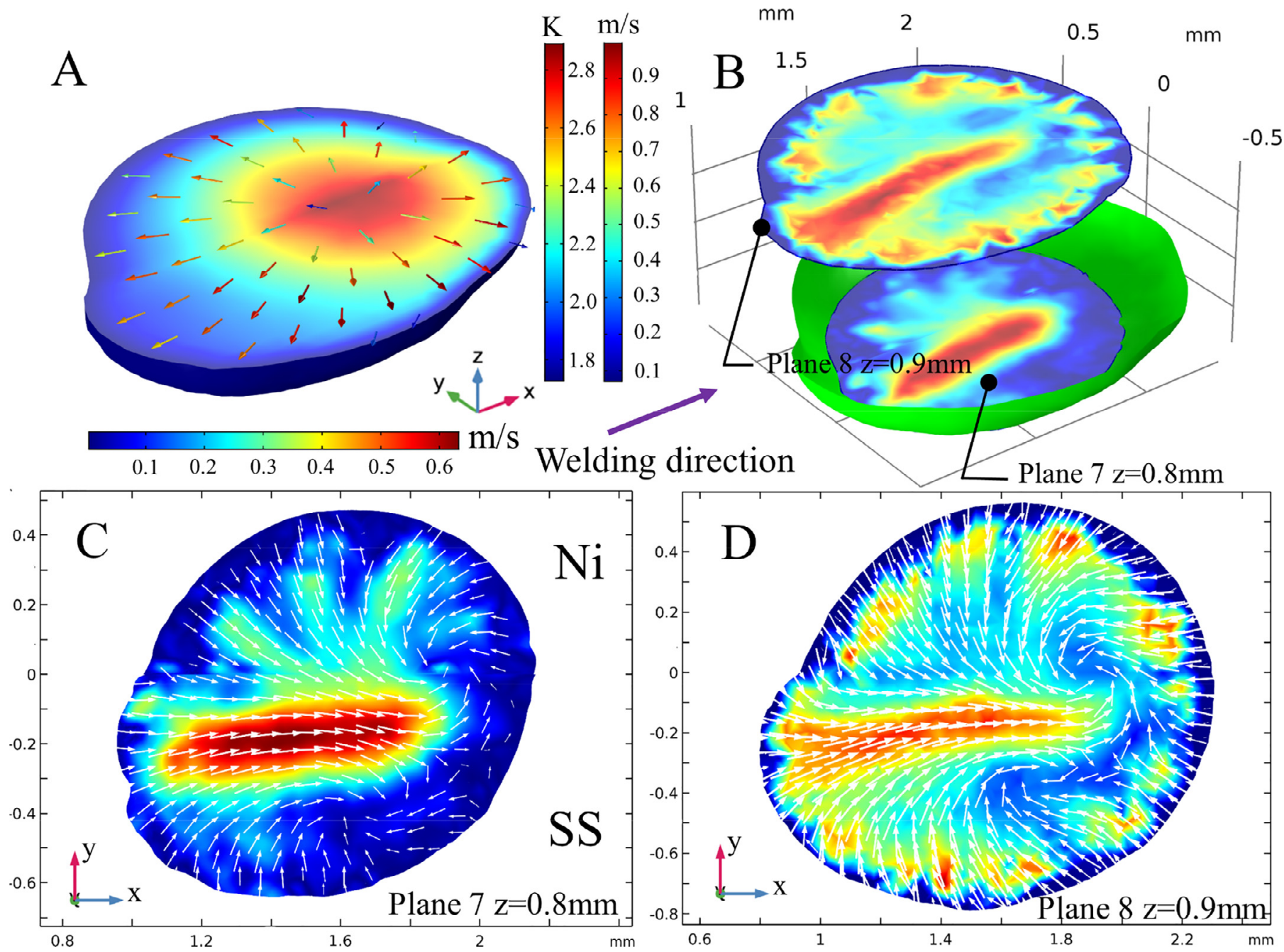

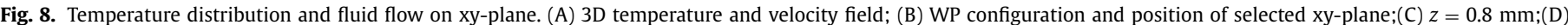
$z=0.9 \mathrm{~mm}$.

Table 4

Case study of different surface tension temperature coefficient of SS and $\mathrm{Ni}$.

\begin{tabular}{lllll}
\hline & Case1 & Case2 & Case3 & Case4 \\
\hline $\mathrm{SS}$ & - & + & + & - \\
$\mathrm{Ni}$ & + & - & + & - \\
\hline
\end{tabular}

sion (TCST) will change its sign from positive to negative in certain situation, which has a great effect on Marangoni convection. For example, the sign transition of TCST from negative to positive will occur at a certain time when the sulfur content is relatively higher in the WP [21]. Because the mass transfer is dominated by convection, the sign transition mentioned above is thus expected to have nonnegligible effect on mass transfer. Therefore, four study cases listed in Table 4 including negative TCST and positive TSCT are performed to investigate the effect of TCST on mass transfer. For these 4 cases, with the absolute value of TCST (listed in Table 2) unchanged, the positive sign represents that the sign of TCST for 304SS or Ni is positive, and the negative sign denotes that the sign of TCST for 304SS or Ni is negative. For the situation of case 4, both the TCST of 304SS and Ni are negative, which is consistent with the situation discussed in above sections. For simplification, the details of fluid flow and mass transfer for case 4 are not listed in Fig. 15. The velocity field and $\mathrm{Ni}$ element distribution for case 1 , case 2 and case 3 are illustrated from Fig. 15A to $C$ respectively. Compared with other situations, the WP depth increases a lot when the TCST of SS changes from negative to positive (see case 2 and case 3 ). In conclusion, the TCST of SS has much more important influence on the fluid flow and mass transfer process compared with the TCST of Ni. From Fig. 15A to C, it is observed that Ni is more uniformly mixed in case 1 and case 2 than that in case 3 and case 4 . Besides, the Ni distribution is relatively uniform in case 1 and case 2 when the TCST changes its sign between two sides. Consequently, it could be concluded that the TCST has a significant effect on the mass transportation of dissimilar couple, and the concentration is more uniformly diluted when the transition from negative to positive or from positive to negative occurs between two substrates.

In addition, sulfur in the substrate [21,34] and oxygen in the environment atmosphere [33,34] are all proved to be related to the sign transition of TCST. Therefore, element concentration control could be realized to a certain level through the adjustments of dilution rate of the WP and the protective gas flow rate by optimizing welding parameters, which will be discussed in future studies.

As mentioned above, the formation of UMZ has largely affected the joint properties and makes it susceptible to defects such as cracks and failures, and the study of UMZ is still a serious challenging problem in dissimilar welding and additive manufacturing. The viscosity of UMZ changes so many times and fluid flow is restricted in this area, but the density, effective heat conductivity 

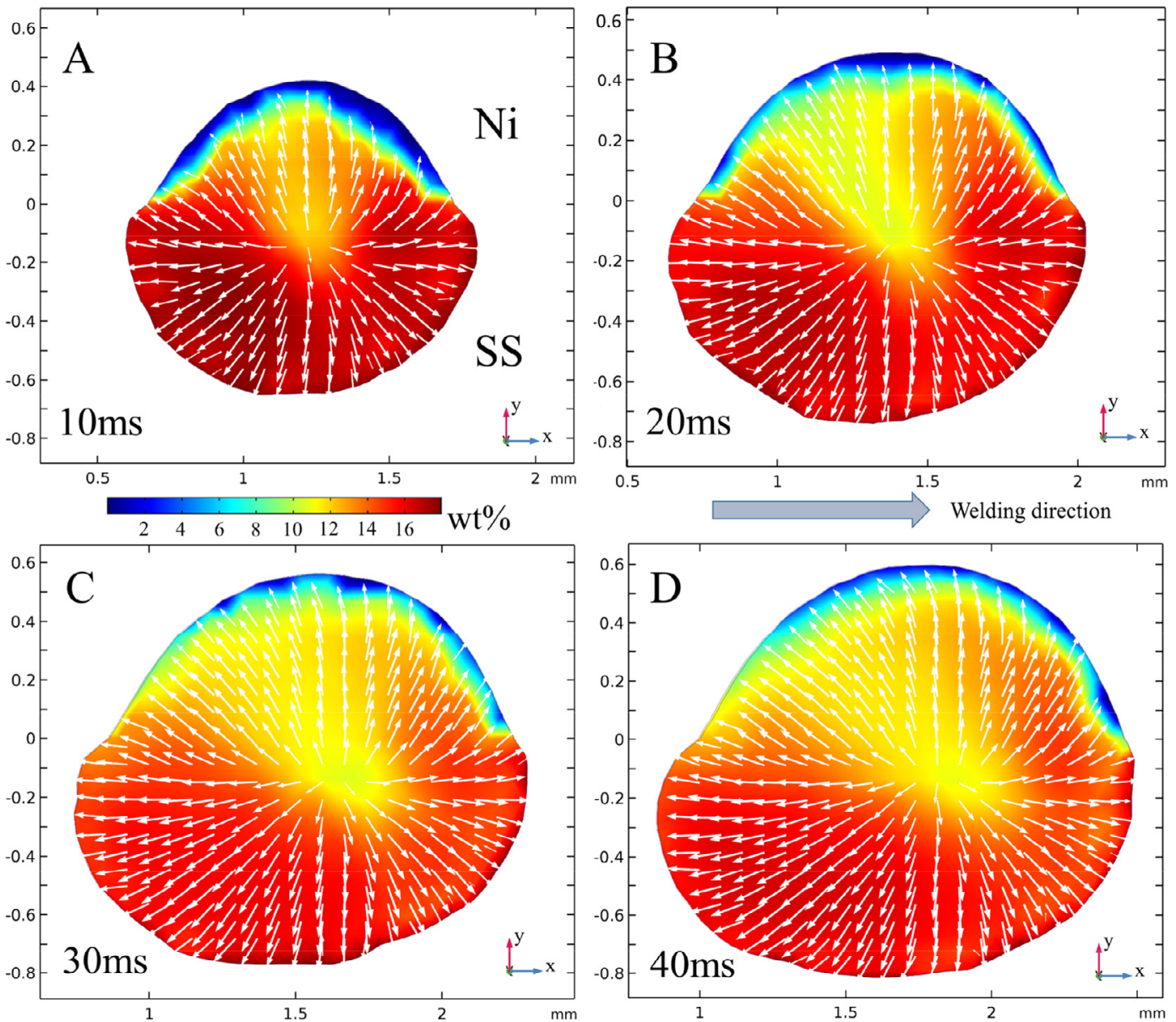

Fig. 9. Cr element distribution at different welding times;(A) $10 \mathrm{~ms}$; (B) $20 \mathrm{~ms}$; (C) $30 \mathrm{~ms}$; (D) $40 \mathrm{~ms}$.
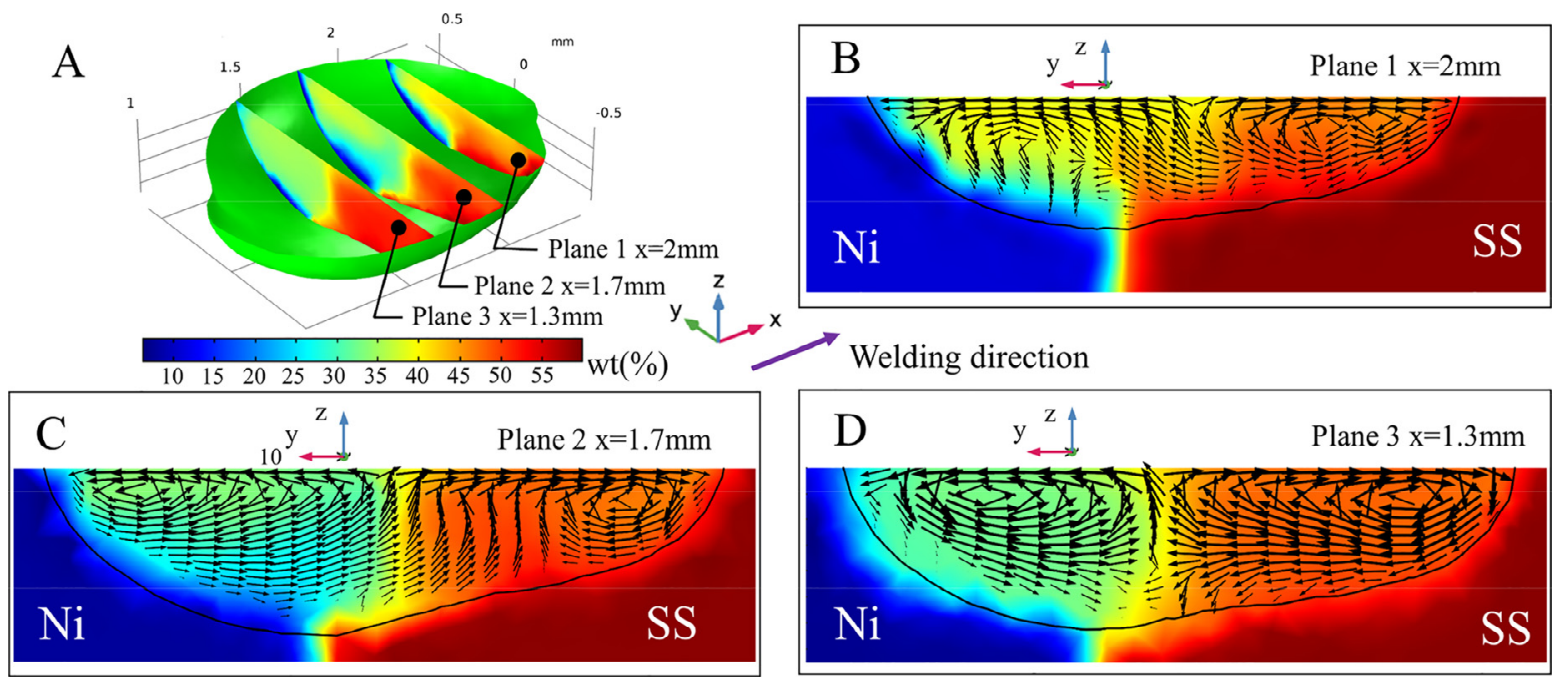

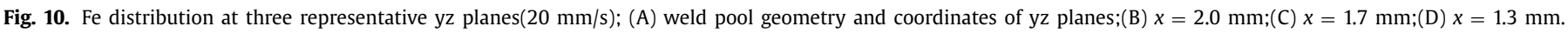

and specific heat of UMZ are on the same order compared with the properties of the mixture inside the melt pool [31,32]. Therefore, the increase of dynamic viscosity is expected to have more obvious effect on the fluid flow and mass transfer in this area. To fundamentally comprehend its effect, velocity field and Ni element distribution under different dynamic viscosity are analyzed. As illustrated in Fig. 16, two situations where the viscosity of SS and Ni are 10 times and 100 times of the normal viscosity, respectively, are simulated and compared with the normal situation. The heat transfer Peclet number, $\mathrm{Pe}_{\mathrm{T}}$, which is defined as the ratio of the effect of heat convection and heat conduction [6], decreases with the increase of dynamic viscosity, which is on the order of 10 for $10 \mathrm{x}$ and 1 for $100 \mathrm{x}$ situation, respectively. As a result, the effect of heat conduction becomes more and more important in the change from normal viscosity to $10 \mathrm{x}$ and $100 \mathrm{x}$ situation, and the WP dimensions increase with a nearly circular configuration at each side. 

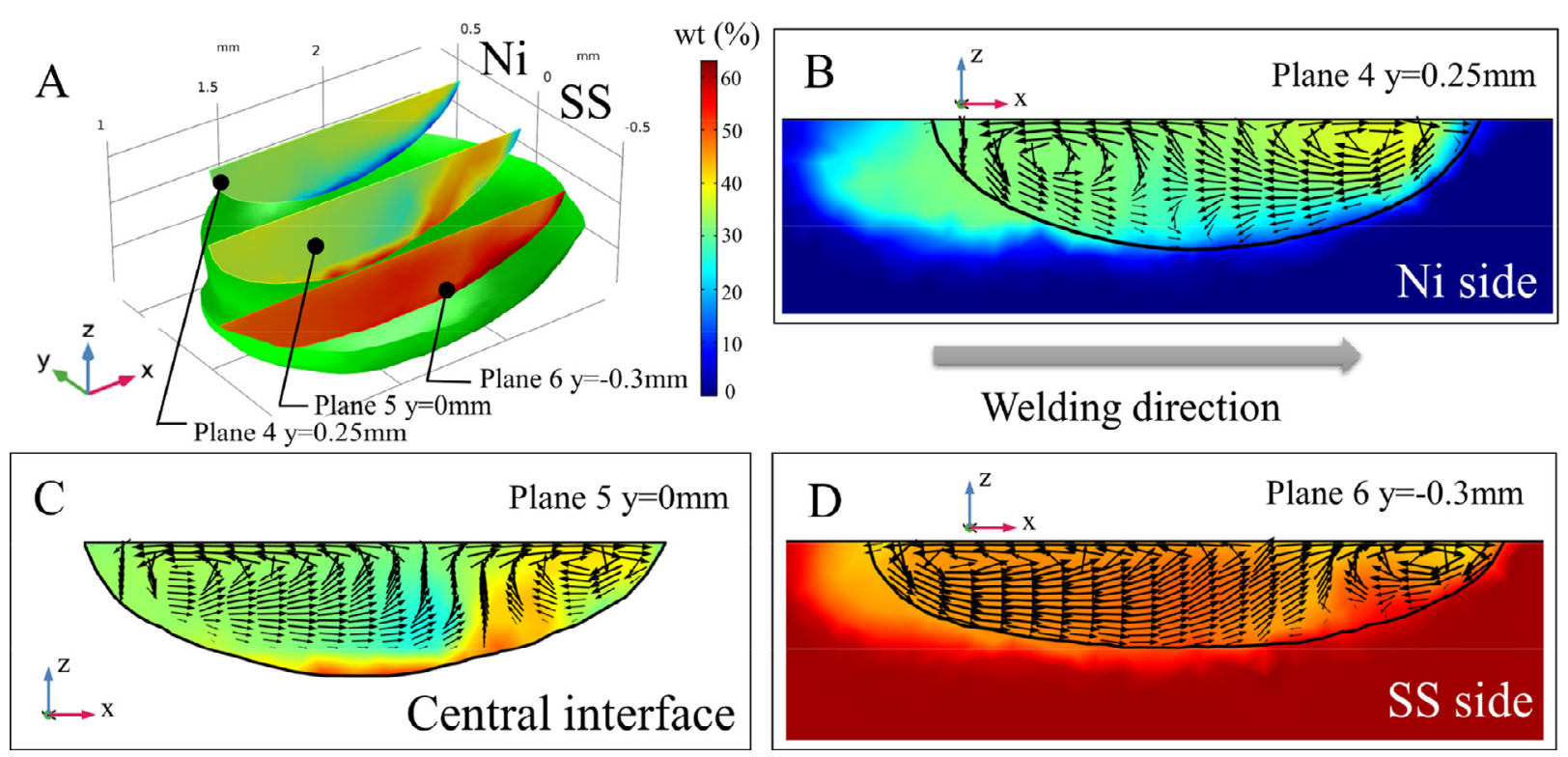

Fig. 11. Fe distribution at three representative $x z$ planes $(20 \mathrm{~mm} / \mathrm{s})$; (A) weld pool geometry and coordinates of $\mathrm{xz}$ planes;(B) $y=0.25 \mathrm{~mm}$;(C) $y=0$;(D) $y=-0.3 \mathrm{~mm}$.

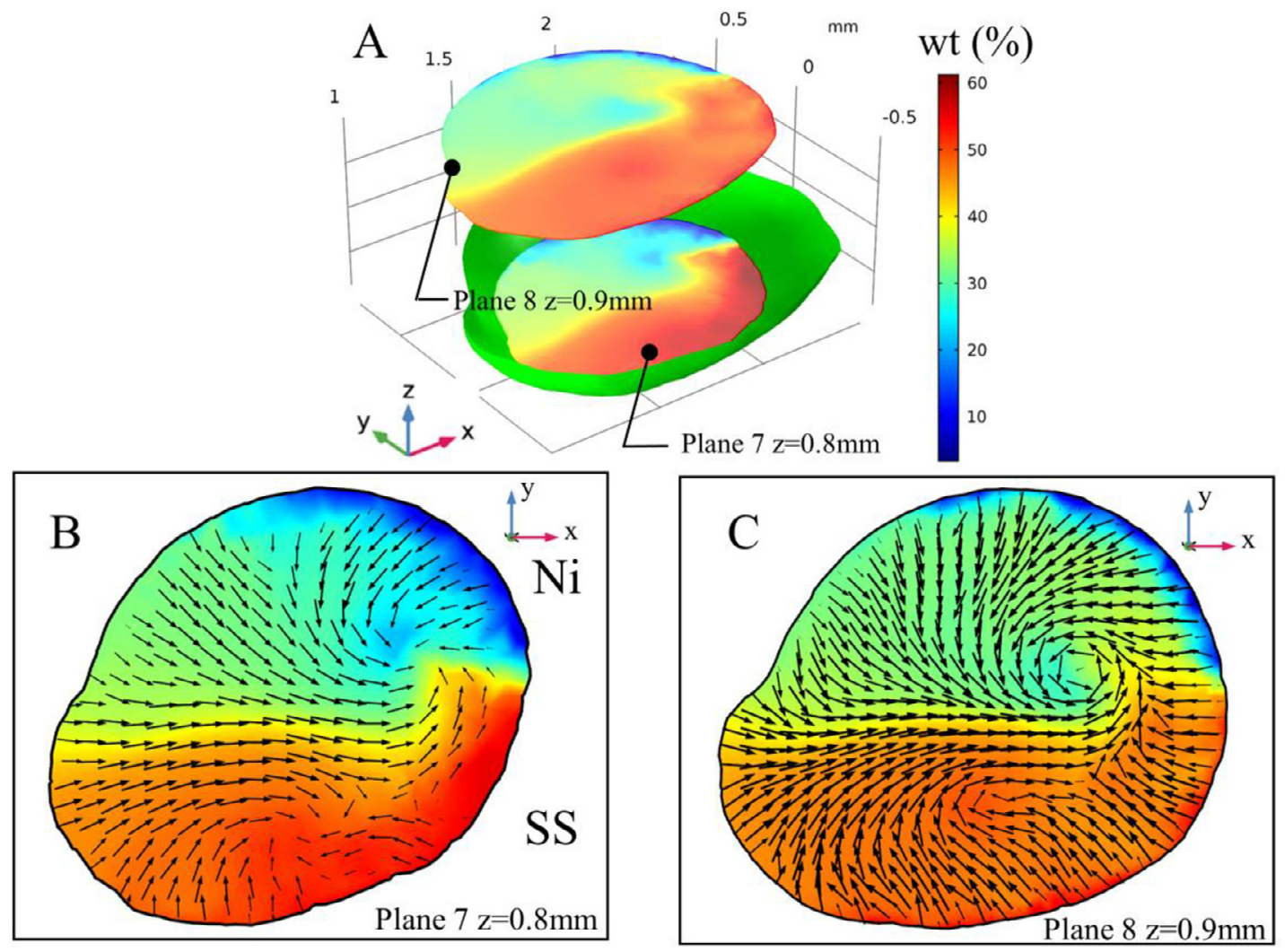

Fig. 12. Fe distribution at two representative xy planes $(20 \mathrm{~mm} / \mathrm{s}) ;(\mathrm{A})$ weld pool geometry and coordinates of $\mathrm{xy}$ planes;(B) $z=0.8 \mathrm{~mm}$;(C) $z=0.9 \mathrm{~mm}$.

Compared to Fig. 9, the WP length decreases, and fluid flow becomes weaker when dynamic viscosity increases. The $\mathrm{Pe}_{\mathrm{c}}$ is calculated to be on the order of $10^{2}$ and 10 for the $10 \mathrm{x}$ and $100 \mathrm{x}$ situation, respectively. As a result, the convection becomes weaker and alloy elements ( $\mathrm{Ni}$, for example) are much more nonuniformly mixed. Additionally, the solidification time will be shortened with the increase of viscosity, because the length of the WP becomes smaller, but the welding speed is kept constant. The shorter solidification time is also not benefit for the uniform dilution inside the WP.
The effects of TCST and dynamic viscosity on fluid flow and mass transfer are discussed here and validated to be nonnegligible in the WP study of welding process. The transition of TCST from negative to positive mentioned above is commonly found in welding and additive manufacturing and imposes great effects on heat and mass transfer. Besides, the fundamental study of the fluid flow and mass transfer in the situations of $10 x$ and $100 x$ normal dynamic viscosity reveals that fluid flow is seriously restricted and species are much more nonuniformly diluted in the UMZ, which also provides a basic understanding for the formation of UMZ. 
Table 5

Comparison of heat and mass transfer between laser linear welding and laser spot welding.

\begin{tabular}{llllll}
\hline Process & Peak temperature(K) & Peak velocity $(\mathrm{m} / \mathrm{s})$ & $P_{e T}$ & $P_{e C}$ & Mixing time (ms) \\
\hline Linear & 2920 & 1.27 & 116.2 & 1430 & 78 \\
Spot & 3000 & 1.51 & 137.3 & 1700 & 205 \\
\hline
\end{tabular}

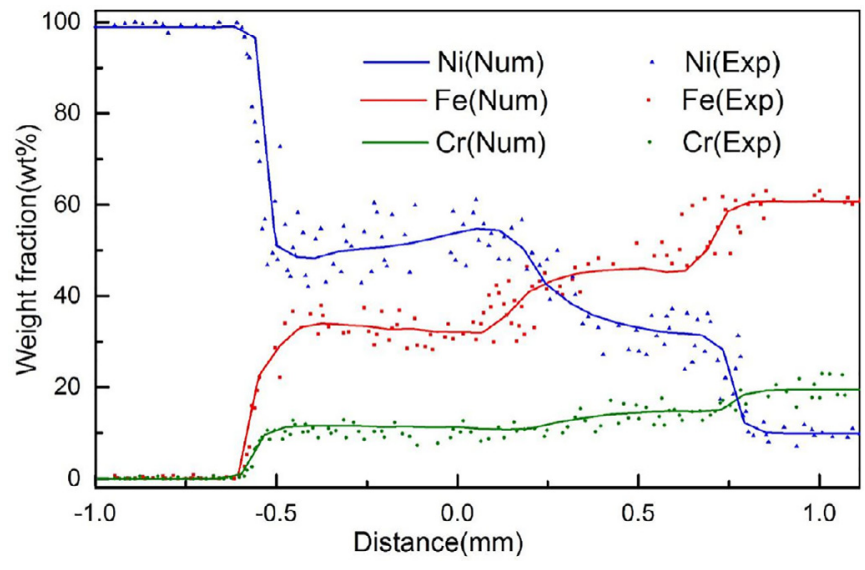

Fig. 13. Comparison of the calculated element distribution and EDS result.

\subsubsection{Comparison with the laser spot welding}

Heat and mass transfer are sensitive to welding speed [32], which is also a vital processing parameter for linear welding. Actually, when the welding speed approaches infinitely small, the situation for linear welding is similar to that for spot welding. For further understanding of the effect of welding speed, some comparisons between these two welding methods are carried out. It should be noted that the comparisons here are mainly aimed at comprehending the differences caused by these two welding methods. For this purpose, the processing parameters for spot welding are set as similar as those for linear welding as possible. In detail, for spot welding, the laser power is $800 \mathrm{~W}$ and the laser beam radius is $0.57 \mathrm{~mm}$, which are all same as the parameters used for linear welding in above simulation. Besides, the welding speed and duration time for linear welding and spot welding are $20 \mathrm{~mm} / \mathrm{s}$ and $200 \mathrm{~ms}$, respectively, which results in similar WP dimensions (see Fig. 17) and makes the comparison reasonable. As shown in Table 5 , the peak temperature is $3000 \mathrm{~K}$ for spot welding and $2920 \mathrm{~K}$ for linear welding. The heat transfer Peclet number is 117.5 for linear welding and 137.3 for spot welding, indicating that heat transfer processes for them are similar and both are dominated by heat convection [6], but convective heat transfer for spot welding is a little stronger.

Based on former discussion, the comparison regarding mass transfer is then carried out. As illustrated in Table $5, \mathrm{Pe}_{\mathrm{C}}$ is on the order of $10^{3}$ and it is a little larger for spot welding, indicating that mass transfer processes for them are both dominated by convection [9]. However, resulting from the high speed movement of laser source, the melting/solidification process for linear welding is quick, as a result, the mixing time for laser linear welding is nearly 3 times smaller than that for spot welding, resulting in a non-uniform dilution due to the non-sufficient mixing time. For a more illustrative comparison, the $\mathrm{Cr}$ distribution at top surface for linear welding and spot welding are plotted and compared together in Fig. 17, and $\mathrm{Cr}$ distribution is obviously more uniform for spot welding. Besides, the melt pool dimensions obtained from these two welding methods are similar, which is also the basic for the comparison presented here. In conclusion, the difference of dilution between these two methods is mainly caused by mixing time, which is observed longer for spot welding. A more uniform dilution is thus expected for a longer mixing time. Besides, the non-uniform mixing contributes to the formation of defects in dissimilar welding, such as the liquid cracking [31]. Strategies that may obtain larger WP dimension and longer mixing time, such as higher power input and lower welding speed [32], could be used to obtain more uniform dilution and high-quality joining.

Finally, the solidification behavior is characterized through the comparison of solidification parameters. As illustrated in Table 6, though their WP dimensions are similar, both the temperature gradient and solidification rate at the welding center line are larger for spot welding. In detail, the temperature gradient for spot welding is nearly 1.5 times larger, and the solidification rate for spot

Table 6

Comparison of solidification parameters at the welding center line between laser linear welding and laser spot welding.

\begin{tabular}{lll}
\hline Process & Linear & Spot \\
\hline Temperature gradient G $(\mathrm{K} / \mathrm{mm})$ & 1749 & 2571 \\
Solidification rate $\mathrm{R}(\mathrm{mm} / \mathrm{s})$ & 20.0 & 61.2 \\
Cooling rate GR $(\mathrm{K} / \mathrm{ms})$ & 35.0 & 157.4 \\
Morphology parameter G/R $\left(\mathrm{K} \bullet \mathrm{s} / \mathrm{mm}^{2}\right)$ & 87.5 & 42.0
\end{tabular}
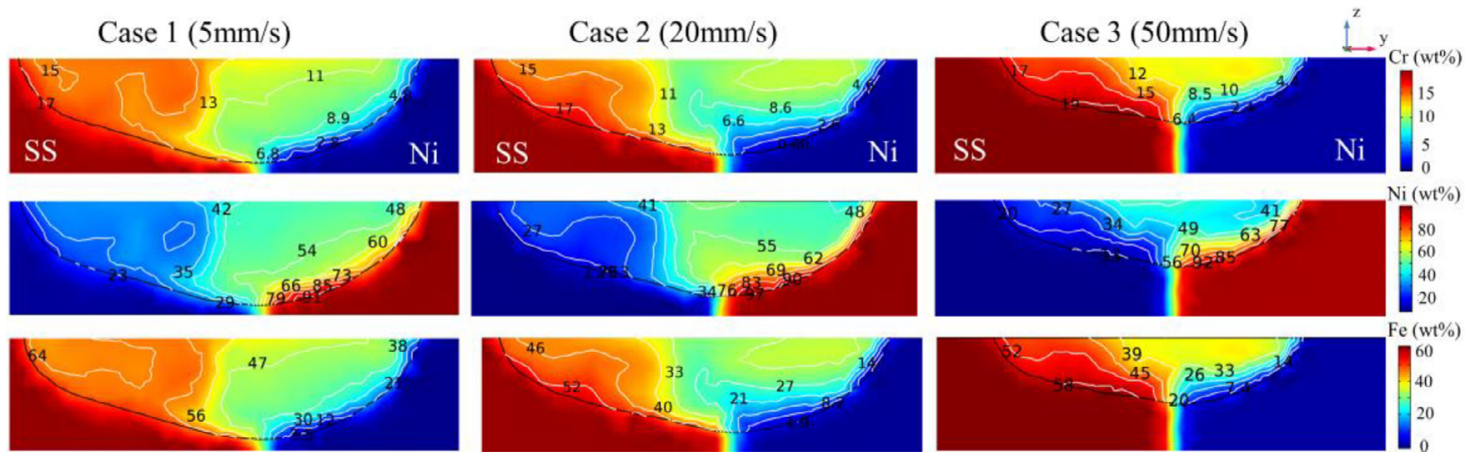

Fig. 14. Alloy element ( $\mathrm{Cr}$, Fe and $\mathrm{Ni})$ distribution on the cross section under different welding speed. 

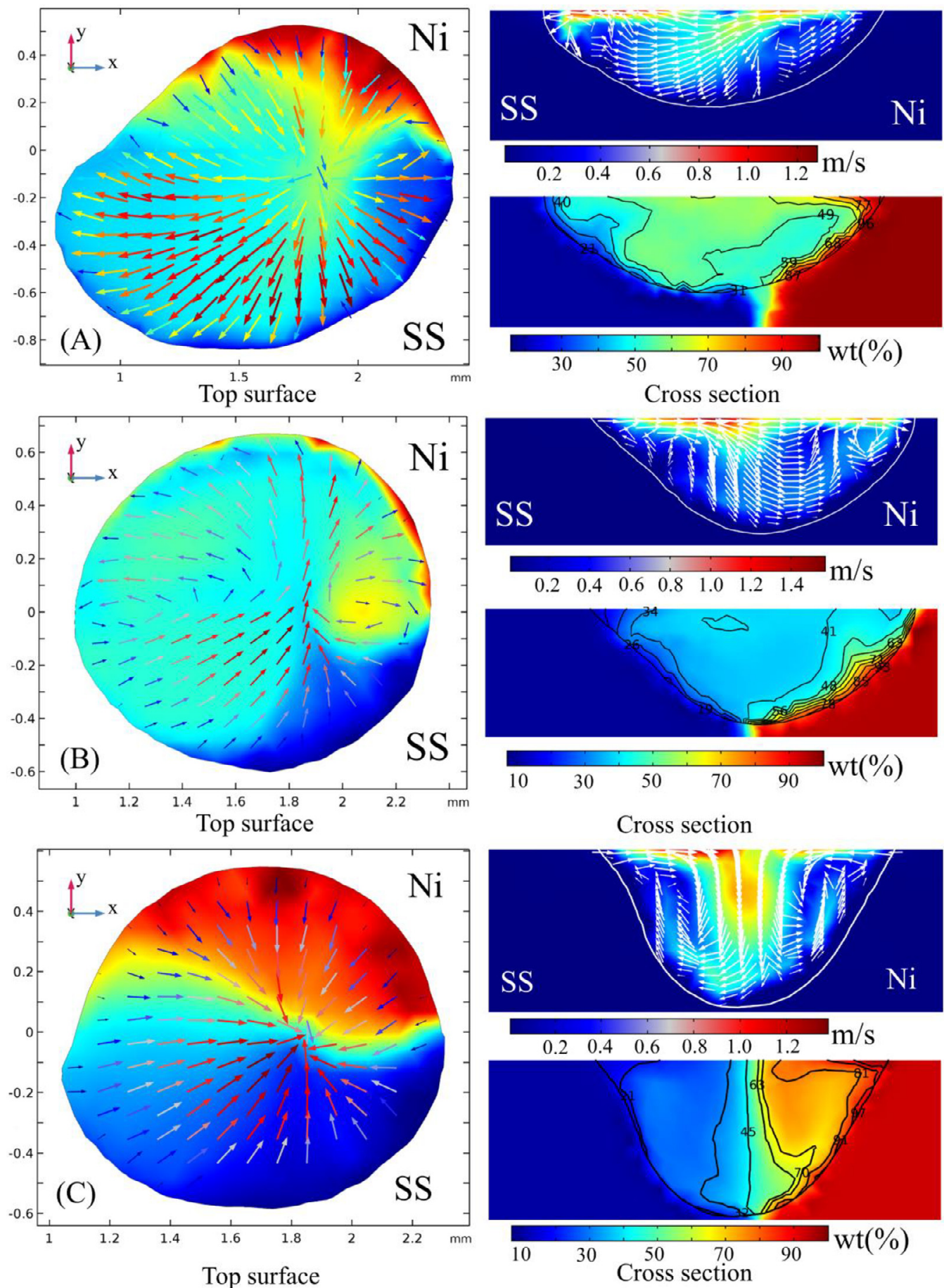

Fig. 15. Velocity field and Ni element distribution under different surface tension temperature coefficients.

welding is almost 3 times larger, indicating that the effect of solidification rate on final microstructure is more obvious. As a result, cooling rate for spot welding is almost 4.5 folds larger and morphology parameter for spot welding is 2.1 folds smaller. Consequently, based on the discussion and conclusion at thermal behavior part, the dendrite grains and smaller microstructure scale maybe more prone to occurring in spot welding [3,6].

It should be noted that the conclusions in this part indicate aspects of the differences of heat and mass transfer between laser linear welding and laser spot welding, and the results should be used carefully because the comparisons carried out here strongly depend on welding parameters. However, the difference of solidification phenomenon is obvious and it still could be qualitatively concluded that cooling rate GR is larger and morphology parameter $G / R$ is smaller for spot welding, The WP existing time for spot welding is much longer, resulting in a longer mixing time and more uniform dilution. Nevertheless, the obtained conclusions provide a fundamental understanding of heat and mass transfer characteristics by comparing some key parameters for these two welding processes, such as the $P e_{T}$ and $P e_{C}$, which are also benefit for understanding the quality assessment depending on different welding methods. 

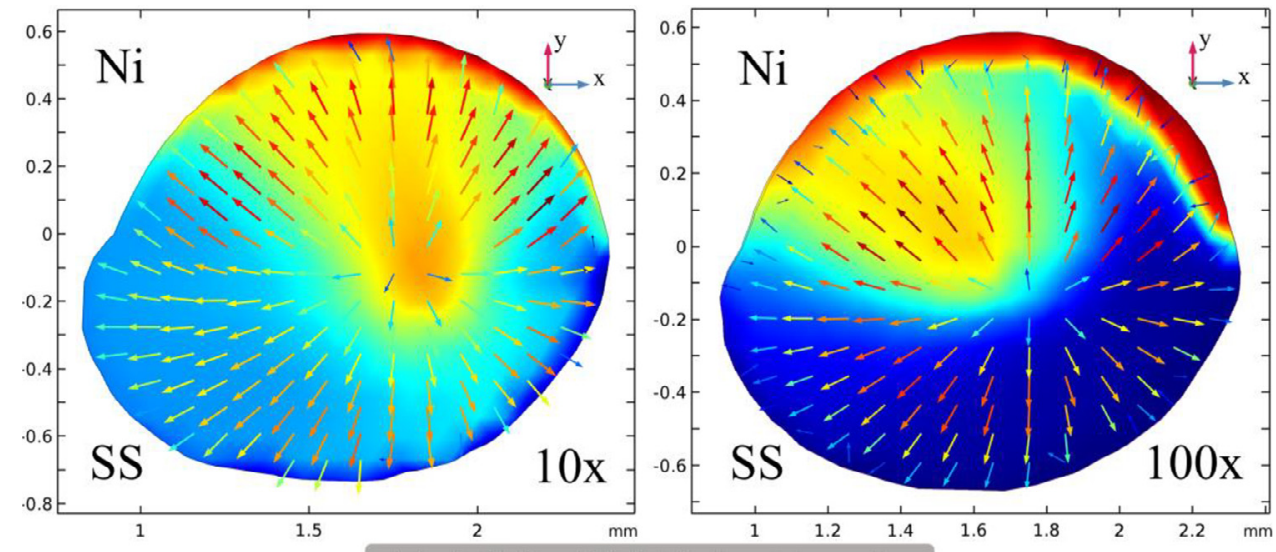

Velocity field and Ni distribution at top surface

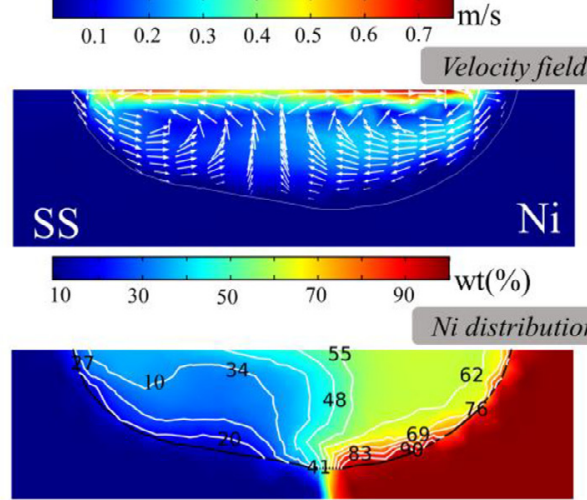

(A) 10x normal viscosity $\begin{array}{lllllllll}0 & 0.02 & 0.04 & 0.08 & 0.1 & 0.12 & 0.14 & 0.16 & \mathrm{~m} / \mathrm{s}\end{array}$
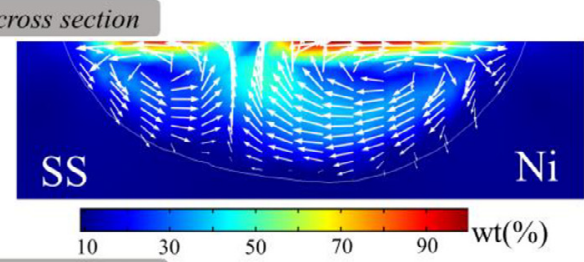

Fig. 16. Velocity field and Ni element distribution under different dynamic viscosity conditions.
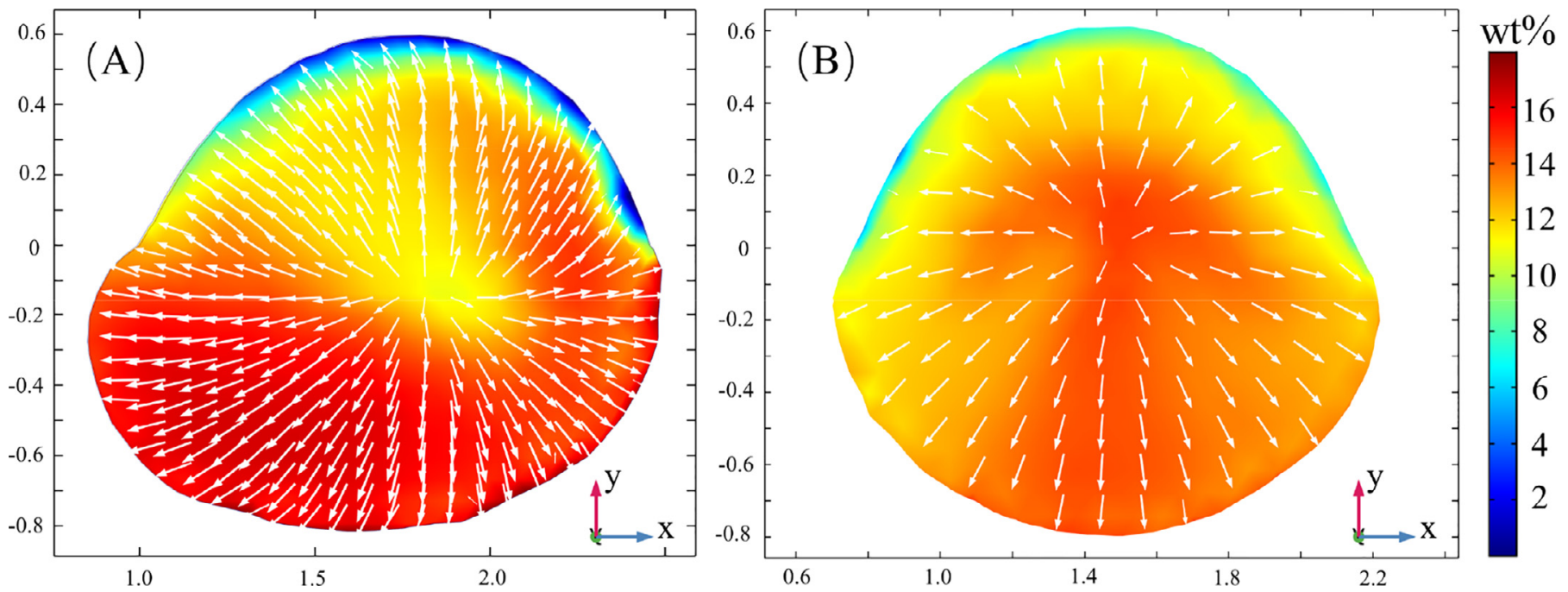

Fig. 17. Cr distribution at top surface from different welding methods. (A) linear welding; (B) spot welding.

\section{Conclusion}

In this work, an improved fluid model was developed to investigate the thermal behavior, solidification characteristics, fluid flow and concentration dilution during laser linear dissimilar welding of SS and Ni. Some basic conclusions could be listed as follows.

(1) The transition from planar to coarser cellular to equiaxed dendrites is observed with the increase of cooling rate GR and the decrease of morphology parameter G/R at SS side. The transition from coarser cellular to fine cellular to equiaxed dendrites is also found at $\mathrm{Ni}$ side. The scale of the solidified microstructure at SS side is finer than that at $\mathrm{Ni}$ side from experimental observation.

(2) The convection at $\mathrm{Ni}$ side is more intense due to the lower dynamic viscosity, and results in a larger vortex. The direction of fluid flow is generally from SS side to Ni side at front of the melt pool but changes from Ni side to SS side at back. The fluid flow is more intense away from the interface of $304 \mathrm{SS}$ and $\mathrm{Ni}$, and its magnitude is larger at upper area. 
(3) $P e_{c}$ is calculated to be on the order of $10^{2}$ for laser linear welding, and convection is thus the main mass transfer mechanism. Elements in SS, such as $\mathrm{Cr}$ and $\mathrm{Fe}$, are mainly mixed at front of the melt pool and then transported to the back, but $\mathrm{Ni}$ is mainly mixed at middle part and transported to front part and rear side. Besides, resulting from the intense fluid flow at upper part, element is mainly mixed at the top area and then transported towards bottom by the 3D vortex flow. There is an obvious redistribution at rear side of the WP, which makes the transported elements, such as $\mathrm{Fe}$ and $\mathrm{Ni}$, more uniformly distributed. The simulated element distribution shows great correspondence with the EDS results.

(4) Resulting from more intense fluid flow and longer mixing time, elements are more uniformly mixed under lower welding speed. Alloy elements are more uniformly mixed when the surface tension temperature coefficient changes its sign from positive to negative, or from negative to positive between 304SS and nickel. Due to intense fluid flow and longer solidification time, decreased dynamic viscosity is benefit for the concentration dilution inside the melt pool.

(5) $P e_{T}$ for laser spot welding and laser linear welding are both on the order of $10^{3}$, indicating that the heat transfer processes for these two methods are similar and both dominated by convection. Larger cooling rate GR and smaller morphology parameter $\mathrm{G} / \mathrm{R}$ are observed in laser spot welding. The difference of dilution between these two methods is mainly caused by mixing time, and the larger mixing time for spot welding results in more uniform dilution.

\section{Declaration of Competing Interest}

All the authors declare that there is no conflict of interest.

\section{CRediT authorship contribution statement}

Zhiyong Li: Conceptualization, Investigation, Data curation, Methodology, Validation, Visualization, Writing - original draft, Writing - review \& editing. Gang Yu: Supervision, Project administration, Funding acquisition, Writing - review \& editing, Resources. Xiuli He: Supervision, Funding acquisition, Project administration, Resources. Shaoxia Li: Validation, Visualization. Zixun Li: Validation, Conceptualization.

\section{Acknowledgments}

This work is supported by the National Natural Science Foundation of China under grant number 11672304. Besides, the authors thank for the Beijing Municipal Commission of Science and Technology (Z181100003818015).

\section{Reference}

[1] S. Chakraborty, S. Mukherjee, R. Galun, Y. Estrin, I. Manna, Transport phenomena in conduction mode laser beam welding of Fe-Al dissimilar couple with Ta diffusion barrier, Int. J. Heat Mass Transf. 53 (23-24) (2010) 5274-5282.

[2] M. Bayat, S. Mohanty, J.H.H. Aidun, A systematic investigation of the effects of process parameters on heat and fluid flow and metallurgical conditions during laser-based powder bed fusion of Ti6Al4V alloy, Int. J. Heat Mass Transf. 139 (2019) 213-230.

[3] Z. Li, G. Yu, X. He, S. Li, C. Tian, B. Dong, Analysis of surface tension driven flow and solidification behavior in laser linear welding of stainless steel, Opt. Laser Technol. 123 (2020) 105914

[4] J.P. Oliveira, Z. Zeng, C. Andrei, F.M. Braz Fernandes, R.M. Miranda, A.J. Ramirez, T. Omori, N. Zhou, Dissimilar laser welding of super elastic NiTi and CuAlMn shape memory Alloys, Mater. Des. 128 (2017) 166-175.

[5] A. Artinov, M. Bachmann, M. Rethmeier, Equivalent heat source approach in a 3D transient heat transfer simulation of full-penetration high power laser beam welding of thick metal plates, Int. J. Heat Mass Transf. 122 (2018) 1003-1013.
[6] Z.Y. Li, G. Yu, X.L. He, Study of thermal behavior and solidification characteristics during laser welding of dissimilar metals, Results Phys. 12 (2019) $1062-1072$.

[7] Y.W. Hu, X.L. He, G. Yu, S.S. Zhao, Capillary convection in pulsed butt welding of miscible dissimilar couple, J. Mech. Sci. Technol. 231 (13) (2016) 24292440.

[8] X. Chen, G. Yu, X. He, S. Li, Z. Li, Numerical study of heat transfer and solute distribution in hybrid laser-MIG welding, Int. J. Therm. Sci. 149 (2020) 106182.

[9] Y. Hu, X. He, G. Yu, Z. Ge, C. Zheng, W. Ning, Heat and mass transfer in laser dissimilar welding of stainless steel and nickel, Appl. Surf. Sci. 2012 (258) (2012) 5914-5922

[10] L. Cao, D. Liu, P. Jiang, X. Shao, Q. Zhou, Y. Wang, Multi-physics simulation of dendritic growth in magnetic field assisted solidification, Int. J. Heat Mass Transf. 144 (2019) 118673.

[11] A. Traidia, F. Roger, Numerical and experimental study of arc and weld pool behavior for pulsed current GTA welding, Int. J. Heat Mass Transf. 54 (9-10) (2011) 2163-2179.

[12] X.Y. Chen, G. Yu, X.L. He, S.X. Li, H.B. Miao, Effect of droplet impact on molten pool dynamics in hybrid laser-MIG welding of aluminum alloy, Int. J. Adv. Manuf. Technol. 96 (2018) 209-222.

[13] L. Li, G. Peng, J. Wang, J. Gong, S. Meng, Numerical and experimental study on keyhole and melt flow dynamics during laser welding of aluminium alloys under subatmospheric pressures, Int. J. Heat Mass Transf. 133 (2019) 812 826.

[14] R. Zhang, X. Tang, L. Xu, F. Lu, H. Cui, Study of molten pool dynamics and porosity formation mechanism in full penetration fiber laser welding of Al-alloy, Int. J. Heat Mass Transf. 148 (2020) 119089.

[15] X. He, P.W. F.uerschbach, T. DebRoy, Heat transfer and fluid flow during laser spot welding of 304 stainless steel, J. Phys. D Appl. Phys. 36 (2003) 13881398.

[16] A. Traidia, F. Roger, Numerical and experimental study of arc and weld pool behavior for pulsed current GTA welding, Int. J. Adv. Manuf. Technol. 54 (2011) 2163-2179.

[17] A. Bahrami, D.T Valentine, B.T. Helenbrook, D.K. Aidun, Study of mass transport in autogenous GTA welding of dissimilar metals, Int. J. Heat Mass Transf. 85 (2015) 41-53.

[18] Z.Y. Li, G. Yu, X.L. He, Numerical and experimental investigations of solidification parameters and mechanical property during laser dissimilar welding, Met. Basel 8 (10) (2018) 799.

[19] J.P. O.liveira, K. Ponder, E. Brizes, T. Abke, P. Edwards, A.J. Ramirez, Combining resistance spot welding and friction element welding for dissimilar joining of aluminum to high strength steels, J. Mater. Process. Technol. 273 (2019) 116192

[20] N. Jimenez, A. Mena, P.J. Simar, Jacques On the interplay between intermetallic controlled growth and hot tearing susceptibility in Al-to-steel welding with additional interlayers, Mater. Des. 180 (2019) 107958.

[21] Z.T. Gan, G. Yu, X.L. He, S.X. Li, Surface-active element transport and its effect on liquid metal flow in laser assisted additive manufacturing, Int. Commun. Heat Mass 86 (2017) 206-214.

[22] H.L. Wei, J.W. Elmer, T. DebRoy, Origin of grain orientation during solidification of an aluminum alloy, Acta Mater. 115 (2016) 123-131.

[23] Z.T. Gan, G. Yu, X.L. He, S.X. Li, Numerical simulation of thermal behavior and multicomponent mass transfer in indirect laser deposition of Co-base ally on steel, Int. J. Heat Mass Transf. 104 (2017) 28-38.

[24] A. Bahrami, B.T. Helenbrook, D.T. Valentine, D.K. Aidun, Fluid flow and mixing in linear GTA welding of dissimilar ferrous alloy, Int. J. Heat Mass Transf. 93 (2016) 729-741.

[25] A. Artinov, M. Bachmann, M. Rethmeier, Equivalent heat source approach in a $3 \mathrm{D}$ transient heat transfer simulation of full-penetration high power laser beam welding of thick metal plates, Int. J. Heat Mass Transf. 122 (2018) 1003-1013.

[26] L. Li, G. Peng, J. Wang, J. Gong, S. Meng, Numerical and experimental study on keyhole and melt flow dynamics during laser welding of aluminum alloys under subatmospheric pressures, Int. J. Heat Mass Transf. 133 (2019) 812826.

[27] R. Zhang, X. Tang, L. Xu, F. Lu, H. Cui, Study of molten pool dynamics and porosity formation mechanism in full penetration fiber laser welding of Al-alloy, Int. J. Heat Mass Transf. 148 (2020) 119089.

[28] V. Voller, C. Prakash, A fixed grid numerical modelling methodology for convection-diffusion mushy region phase-change problems, Int. J. Heat Mass Transf. 30 (8) (1987) 1709-1719.

[29] A.D. Brent, V.R. Voller, K.J. Reid, Eethalphy-Porosity technology for modeling convection-diffusion phase change: application to the melting of a pure metal Numer. Heat Transf. 13 (3) (1988) 297-318.

[30] L. Cao, D. Liu, P. Jiang, X. Shao, Q. Zhou, Y. Wang, Multi-physics simulation of dendritic growth in magnetic field assisted solidification, Int. J. Heat Mass Transf. 144 (2019) 118673.

[31] S. Kou, Welding Metallurgy, John Wiley \& Sons, New York, 2003.

[32] F. Hejripour, B.T. Helenbrook, D.T. Valentine, Daryush K. Aidun, Mass transport and solidification phenomenon in dissimilar metals arc welding, Int. J. Heat Mass Transf. 144 (2019) 118703.

[33] P. Sahoo, T. Debroy, M.J. McNallan, Surface tension of binary metal-surface active solute systems under conditions relevant to welding metallurgy, Metall. Trans. B 19 (1988) 483-491.

[34] Y. Hu, X. He, G. Yu, S. Li, C. Zheng, W. Ning, Experimental and numerical study on laser keyhole welding of $42 \mathrm{CrMo}$ under air and argon atmosphere, Int. J. Adv. Manuf. Technol. 90 (2017) 3555-3565. 